\title{
A molecular survey of outflow gas: velocity-dependent shock chemistry and the peculiar composition of the EHV gas ${ }^{\star \star \star}$
}

\author{
M. Tafalla ${ }^{1}$, J. Santiago-García ${ }^{1,2}$, A. Hacar ${ }^{1}$, and R. Bachiller ${ }^{1}$ \\ ${ }^{1}$ Observatorio Astronómico Nacional (IGN), Alfonso XII 3, 28014 Madrid, Spain \\ e-mail: m.tafalla@oan.es \\ 2 Instituto de Radioastronomía Milimétrica (IRAM), Avenida Divina Pastora 7, Núcleo Central, 18012 Granada, Spain \\ Received 4 June 2010 / Accepted 15 July 2010
}

ABSTRACT

\begin{abstract}
Context. Bipolar outflows from Class 0 protostars often present two components in their CO spectra that have different kinematic behaviors: a smooth outflow wing and a discrete, extremely high-velocity (EHV) peak.

Aims. To better understand the origin of these two outflow components, we have studied and compared their molecular composition. Methods. We carried out a molecular survey of the outflows powered by L1448-mm and IRAS 04166+2706, two sources with prominent wing and EHV components. For each source, we observed a number of molecular lines towards the brightest outflow position and used them to determine column densities for 12 different molecular species.

Results. The molecular composition of the two outflows is very similar. It presents systematic changes with velocity that we analyze by dividing the outflow in three chemical regimes, two of them associated with the wing component and the other the EHV gas. The analysis of the two wing regimes shows that species like $\mathrm{H}_{2} \mathrm{CO}$ and $\mathrm{CH}_{3} \mathrm{OH}$ favor the low-velocity gas, while $\mathrm{SiO}$ and $\mathrm{HCN}$ are more abundant in the fastest gas. This fastest wing gas presents strong similarities with the composition of the "chemically active" L1157 outflow (whose abundances we re-evaluate in an appendix). We find that the EHV regime is relatively rich in O-bearing species compared to the wing regime. The $\mathrm{EHV}$ gas is not only detected in $\mathrm{CO}$ and $\mathrm{SiO}$ (already reported elsewhere), but also in $\mathrm{SO}, \mathrm{CH}_{3} \mathrm{OH}$, and $\mathrm{H}_{2} \mathrm{CO}$ (newly reported here), with a tentative detection in $\mathrm{HCO}^{+}$. At the same time, the EHV regime is relatively poor in C-bearing molecules like CS and $\mathrm{HCN}$, for which we only obtain weak detections or upper limits despite deep integrations. We suggest that this difference in composition arises from a lower $\mathrm{C} / \mathrm{O}$ ratio in the $\mathrm{EHV}$ gas.

Conclusions. The different chemical compositions of the wing and EHV regimes suggest that these two outflow components have different physical origins. The wing component is better explained by shocked ambient gas, although none of the existing shock models explains all observed features. We hypothesize that the peculiar composition of the EHV gas reflects its origin as a dense wind from the protostar or its surrounding disk.
\end{abstract}

Key words. ISM: jets and outflows - ISM: abundances - stars: formation - ISM: molecules

\section{Introduction}

Outflows from the youngest protostars often display the simplest geometry and the highest degree of symmetry, and they are expected to reflect more faithfully than other outflows the properties of the still-mysterious driving wind (e.g., Bachiller \& Tafalla 1999). Among the youngest outflows known, one group stands out for its pristine appearance in maps and the presence in their $\mathrm{CO}$ spectra of distinct extremely high velocity (EHV) features. These features are secondary emission peaks at the highest outflow velocities that tend to appear symmetrically placed from the driving source along the outflow axis. The prototype of this group of outflows is the one driven by the $9 L_{\odot}$ Class 0 protostar L1448-mm, located in the Perseus molecular cloud and first identified by Bachiller et al. (1990). Molecular observations of the L1448 outflow have shown that the moving material in this system lies in two different components: a pair of conical shells and a highly collimated jet. In the spectra, the emission from the shells appears in the form of a gradual outflow wing, while the emission from the jet arises from the faster EHV feature

^ Based on observations carried out with the IRAM 30 m Telescope. IRAM is supported by INSU/CNRS (France), MPG (Germany) and IGN (Spain).

$\star \star$ Appendices are only available in electronic form at http://www . aanda.org
(Bachiller et al. 1995; Jørgensen et al. 2007; Maury et al. 2010; Hirano et al. 2010).

While the shell of relatively slow gas in the L1448 outflow is similar to what is seen in more evolved outflows like L1551 (e.g., Moriarty-Schieven et al. 1987), and therefore likely composed of accelerated ambient gas, the nature of the EHV component is still mysterious. The symmetry, high speed, and fragmented appearance of the EHV gas initially suggested that it may consist of a collection of molecular "bullets" ejected by the central protostar and traveling along the outflow axis (Bachiller et al. 1990). Free-traveling bullets with the observed internal velocity dispersion of the EHV gas, however, would likely dissipate quickly along their path (Richer et al. 1992), and because of this, a number of alternative interpretations to the EHV gas have been offered. One possibility is that the EHV gas represents shocked ambient material accelerated by an invisible jet, as suggested by observations of $\mathrm{SiO}$ emission (Bachiller et al. 1991) and by numerical simulations of momentum transfer from a jet (Chernin et al. 1994). Alternatively, the EHV features could represent internal shocks along a jet owing to variations in the velocity of ejection, similar to those invoked to explain the chains of $\mathrm{HH}$ objects often seen in optical jets (Raga et al. 1990; Raga \& Cabrit 1993; Dutrey et al. 1997; Reipurth \& Bally 2001).

Recent high angular resolution observations of very young outflows have started to offer new clues to the nature of the 
EHV gas and its relation to the outflow shells. An ideal target for this work has been the outflow from IRAS 04166+2706 (I04166 hereafter), a $0.4 L_{\odot}$ YSO in Taurus that presents a number of similarities with the L1448 outflow, including a shell-plus-jet geometry and bright EHV features (Tafalla et al. 2004b; Santiago-García et al. 2009). High angular resolution observations of the I04166 outflow by Santiago-García et al. (2009) show that the shells and the EHV component have very different kinematics. The low-velocity shells seem to arise from ambient gas accelerated by a wide-angle wind, while the EHV gas presents a sawtooth velocity field that is inconsistent with an origin in shocked ambient material, but is well explained as resulting from a series of internal working surfaces traveling along a collimated jet. The I04166 data, therefore, suggest that the outflow consists of two distinct components having different physical origin.

A number of recent theoretical models have also suggested that outflows consist of multiple components (Shang et al. 2006; Banerjee \& Pudritz 2006; Machida et al. 2008; Tomida et al. 2010). Of particular interest for the study of the EHV gas is the so-called "unified model" of Shang et al. (2006). These authors have modeled the interaction between the wind from a protostar and the surrounding dense gas, and suggested that while the outflow shells originate from ambient gas that has been shockcompressed by a wide-angle wind, the jet-like EHV component represents the central and most collimated part of the protostellar wind itself.

Further testing the possible double-nature of the outflow gas can be done through chemical analysis. The chemical composition of a gas is a sensitive indicator of its thermal history, so if the outflow shell and jet-like components have different physical origins, this should be reflected in the components having different chemical abundances. The chemical composition of the outflow gas has been studied in a number of systems, both towards high mass protostars like Orion-IRc 2 (e.g., Blake et al. 1987) and towards low mass objects like NGC1333-IRAS 4, L1157, or BHR 71 (Blake et al. 1995; Bachiller \& Pérez Gutiérrez 1997; Garay et al. 1998). These studies have shown that the outflow composition is characterized by abundance enhancements of species like $\mathrm{SiO}$ and $\mathrm{CH}_{3} \mathrm{OH}$, which are indicative of shock chemistry (van Dishoeck \& Blake 1998). Previous work, however, has not included the very young outflows that have EHV gas, and as a result, the chemistry of this outflow component has not been surveyed in detail and compared with the composition of the rest of the outflow. To fill this observational gap, and to test the possible double nature of the outflow material, we have carried out a systematic molecular survey of the outflow gas towards the two objects with most prominent EHV components, L1448 and I04166.

\section{Target selection and observations}

The intensity of the EHV feature in high density tracers rarely exceeds $0.1 \mathrm{~K}$ and is often significantly weaker, so a molecular survey of the EHV gas requires integration times often in excess of $1 \mathrm{~h}$ per transition. This strongly limits the number of targets that can be studied during a typical observing run, and for this reason, our molecular survey was targeted towards only two outflow positions, one in L1448 and the other in I04166. These positions were selected for having the brightest $\mathrm{CO}(2-1) \mathrm{EHV}$ component in each outflow, and were determined during a preliminary search along each outflow axis. For the L1448 outflow, we selected the position with offsets $\left(16^{\prime \prime},-34^{\prime \prime}\right)$ with respect to the driving

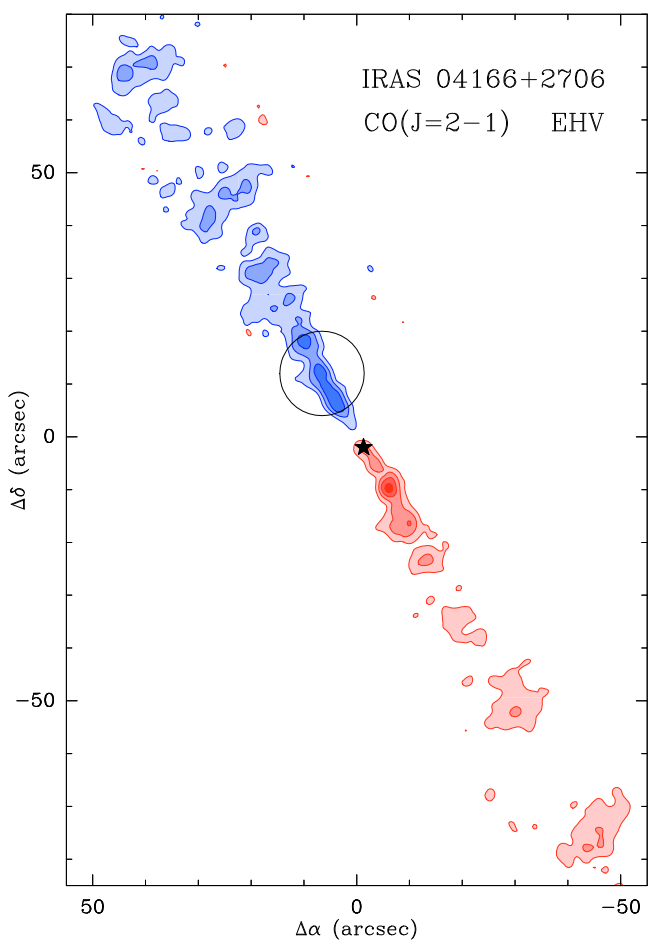

Fig. 1. $\mathrm{CO}(J=2-1)$ map of the extremely high velocity (EHV) component in the I04166 outflow illustrating the location of the molecular survey position with a circle of $16^{\prime \prime}$ diameter (the typical size of the telescope beam width). The CO map is from the Plateau de Bure observations presented in Santiago-García et al. (2009).

source $\left(\alpha(\mathrm{J} 2000)=3^{\mathrm{h}} 25^{\mathrm{m}} 38^{\mathrm{s}} .9, \delta(J 2000)=+30^{\circ} 44^{\prime} 05^{\prime \prime}\right.$; Curiel et al. 1990), which corresponds to the redshifted lobe of the flow. For the I04166 outflow, we selected the position with offsets $\left(8^{\prime \prime}, 14^{\prime \prime}\right)$ with respect to the mm-continuum peak $(\alpha(\mathrm{J} 2000)=$ $4^{\mathrm{h}} 19^{\mathrm{m}} 42^{\mathrm{s}} .5, \delta(\mathrm{J} 2000)=+27^{\circ} 13^{\prime} 36^{\prime \prime}$; Santiago-García et al. 2009), corresponding to the blue lobe of the outflow. To illustrate its location, we present in Fig. 1 the I04166 target position superposed to a high resolution $\mathrm{CO}(2-1)$ map of the EHV gas

In addition to the two survey targets, supplementary positions of the L1448 and I04144 outflows were observed for this project. After detecting bright SO emission in the EHV components of the L1448 and I04166 outflows, we searched for this molecule towards a number of positions along the axis of each flow, to asses whether our target positions were unique in presenting bright $\mathrm{SO}$ and to determine the extent of the SO emission in the EHV gas. Also, in order to derive beam-dilution factors to correct the observed intensities during the multi-line analysis of Sect. 3, we made small $\mathrm{CO}(2-1)$ Nyquist-sampled maps around the two target positions.

All observations presented here were carried out with the IRAM 30 m radio telescope near Granada (Spain) during sessions in 2007 June, 2008 August, and 2009 May-June. A number of receiver configurations was used to maximize the number of molecular lines observed. The list of the main transitions, rest frequencies, and related parameters is given in Table 1. For each line, two spectrometers were used in parallel: the VESPA autocorrelator, configured to provide velocity resolutions of around $1 \mathrm{~km} \mathrm{~s}^{-1}$, and either the $1 \mathrm{MHz}$ filterbank or the wider $(4 \mathrm{MHz})$ WILMA autocorrelator to provide extra frequency coverage. Flat baselines were achieved using wobbler-switching mode, chopping with a frequency of $0.5 \mathrm{~Hz}$ between the source position and a reference position $220^{\prime \prime}$ away in azimuth. The relatively small offset between source and reference position caused some 
Table 1. Main transitions observed ${ }^{1}$

\begin{tabular}{|c|c|c|c|c|}
\hline Transition & $\begin{array}{c}\text { Frequency } \\
(\mathrm{GHz})\end{array}$ & $\begin{array}{c}A_{\mathrm{ul}} \\
\left(\mathrm{s}^{-1}\right) \\
\end{array}$ & $\begin{array}{c}E_{\mathrm{u}} / k \\
(\mathrm{~K}) \\
\end{array}$ & $g_{\mathrm{u}}$ \\
\hline $\mathrm{CO}(J=1-0)$ & 115.3 & $7.20 \times 10^{-8}$ & 5.54 & 3 \\
\hline $\mathrm{CO}(J=2-1)$ & 230.5 & $6.91 \times 10^{-7}$ & 16.6 & 5 \\
\hline${ }^{13} \mathrm{CO}(J=2-1)$ & 220.4 & $6.08 \times 10^{-7}$ & 15.9 & 5 \\
\hline $\mathrm{SiO}(J=2-1)$ & 86.8 & $2.93 \times 10^{-5}$ & 6.26 & 5 \\
\hline $\operatorname{SiO}(J=3-2)$ & 130.3 & $1.06 \times 10^{-4}$ & 12.5 & 7 \\
\hline $\operatorname{SiO}(J=5-4)$ & 217.1 & $5.20 \times 10^{-4}$ & 31.3 & 11 \\
\hline $\operatorname{SiO}(J=6-5)$ & 260.5 & $9.12 \times 10^{-4}$ & 43.8 & 13 \\
\hline $\mathrm{SO}\left(J_{\mathrm{N}}=3_{2}-2_{1}\right)$ & 99.3 & $1.13 \times 10^{-5}$ & 9.23 & 7 \\
\hline $\mathrm{SO}\left(J_{\mathrm{N}}=2_{3}-1_{2}\right)$ & 109.3 & $1.08 \times 10^{-5}$ & 21.1 & 5 \\
\hline $\mathrm{SO}\left(J_{\mathrm{N}}=4_{3}-3_{2}\right)$ & 138.2 & $3.17 \times 10^{-5}$ & 15.9 & 9 \\
\hline $\mathrm{SO}\left(J_{\mathrm{N}}=6_{5}-5_{4}\right)$ & 219.9 & $1.34 \times 10^{-4}$ & 35.0 & 13 \\
\hline $\mathrm{CH}_{3} \mathrm{OH}\left(J_{k}=2_{-1}-1_{-1}\right) \mathrm{E}$ & 96.7 & $2.56 \times 10^{-6}$ & 4.65 & 5 \\
\hline $\mathrm{CH}_{3} \mathrm{OH}\left(J_{k}=2_{0}-1_{0}\right) \mathrm{A}$ & 96.7 & $3.41 \times 10^{-6}$ & 6.97 & 5 \\
\hline $\mathrm{CH}_{3} \mathrm{OH}\left(J_{k}=2_{0}-1_{0}\right) \mathrm{E}$ & 96.7 & $3.41 \times 10^{-6}$ & 12.2 & 5 \\
\hline $\mathrm{CH}_{3} \mathrm{OH}\left(J_{k}=3_{0}-2_{0}\right) \mathrm{E}$ & 145.1 & $1.23 \times 10^{-5}$ & 19.2 & 7 \\
\hline $\mathrm{CH}_{3} \mathrm{OH}\left(J_{k}=3_{-1}-2_{-1}\right) \mathrm{E}$ & 145.1 & $1.10 \times 10^{-5}$ & 11.6 & 7 \\
\hline $\mathrm{CH}_{3} \mathrm{OH}\left(J_{k}=3_{0}-2_{0}\right) \mathrm{A}$ & 145.1 & $1.23 \times 10^{-5}$ & 13.9 & 7 \\
\hline $\mathrm{CH}_{3} \mathrm{OH}\left(J_{k}=5_{-1}-4_{-1}\right) \mathrm{E}$ & 241.8 & $5.81 \times 10^{-5}$ & 32.5 & 11 \\
\hline $\mathrm{CH}_{3} \mathrm{OH}\left(J_{k}=5_{0}-4_{0}\right) \mathrm{A}$ & 241.8 & $6.05 \times 10^{-5}$ & 34.8 & 11 \\
\hline $\mathrm{HC}_{3} \mathrm{~N}(J=10-9)$ & 91.0 & $5.81 \times 10^{-5}$ & 24.0 & 21 \\
\hline $\mathrm{HC}_{3} \mathrm{~N}(J=11-10)$ & 100.1 & $7.77 \times 10^{-5}$ & 28.8 & 23 \\
\hline $\mathrm{HC}_{3} \mathrm{~N}(J=12-11)$ & 109.2 & $1.01 \times 10^{-4}$ & 34.1 & 25 \\
\hline $\mathrm{HC}_{3} \mathrm{~N}(J=16-15)$ & 145.6 & $2.42 \times 10^{-4}$ & 59.4 & 33 \\
\hline $\mathrm{CS}(J=2-1)$ & 98.0 & $1.68 \times 10^{-5}$ & 7.06 & 5 \\
\hline $\mathrm{CS}(J=3-2)$ & 146.7 & $6.07 \times 10^{-5}$ & 14.1 & 7 \\
\hline $\operatorname{CS}(J=5-4)$ & 244.9 & $2.98 \times 10^{-4}$ & 35.3 & 11 \\
\hline $\mathrm{HCO}^{+}(J=1-0)$ & 89.2 & $4.19 \times 10^{-5}$ & 4.28 & 3 \\
\hline $\operatorname{HCN}(J=1-0)$ & 88.6 & $2.41 \times 10^{-5}$ & 4.26 & 3 \\
\hline $\mathrm{H}_{2} \mathrm{CO}\left(J_{K_{\mathrm{a}} K_{\mathrm{c}}}=2_{02}-1_{01}\right)$ & 145.6 & $7.81 \times 10^{-5}$ & 10.5 & 5 \\
\hline $\mathrm{SO}_{2}\left(J_{K_{\mathrm{a}} K_{\mathrm{c}}}=3_{13}-2_{02}\right)$ & 104.0 & $1.01 \times 10^{-5}$ & 7.75 & 7 \\
\hline $\mathrm{H}_{2} \mathrm{~S}\left(J_{K_{\mathrm{a}} K_{\mathrm{c}}}=1_{10}-1_{01}\right)$ & 168.8 & $2.68 \times 10^{-5}$ & 27.9 & 9 \\
\hline $\operatorname{SiS}(J=5-4)$ & 90.8 & $1.19 \times 10^{-5}$ & 13.1 & 11 \\
\hline $\operatorname{HNC}(J=1-0)$ & 90.7 & $2.69 \times 10^{-5}$ & 4.35 & 3 \\
\hline
\end{tabular}

Notes. (1) Molecular parameters from the Cologne Database for Molecular Spectroscopy (http://www.astro.uni-koeln.de/ cdms/), see Müller et al. (2001, 2005).

contamination in the spectra at the ambient cloud velocity, but it did not affect the outflow emission studied here. As a result of the flat baselines, only a zeroth-order polynomial was subtracted from the spectra in the off-line data reduction.

During the observations, the pointing was checked and corrected by making cross scans on nearby continuum sources approximately every two hours, and it was usually below $3^{\prime \prime}$ rms. A number of larger pointing excursions occurred during the 2008 run, and observations with suspicious pointing errors were discarded. Calibration was achieved by observing every $10 \mathrm{~min}$ a sequence of blank sky, an absorber at room temperature, and a cold load, and the calibrated $T_{\mathrm{A}}^{*}$ scale from the telescope was converted into the $T_{\mathrm{mb}}$ scale using the standard beam efficiency factors provided by the telescope system. Further reduction and analysis of the data was carried out using the GILDAS software (http: //wWw . iram. fr/IRAMFR/GILDAS).

\section{Molecular survey results}

\subsection{CO data: wing and EHV regimes}

Figure 2 shows the $\mathrm{CO}(1-0), \mathrm{CO}(2-1)$ and ${ }^{13} \mathrm{CO}(2-1)$ spectra towards the two target positions of our survey. As can be seen, each $\mathrm{CO}$ spectrum presents a broad wing component that starts at the ambient cloud velocity $\left(V_{\mathrm{LSR}}=5.0 \mathrm{~km} \mathrm{~s}^{-1}\right.$ in L1448 and $6.7 \mathrm{~km} \mathrm{~s}^{-1}$ in I04166) and extends towards the red in the case of L1448 and towards the blue in I04166. This wing component decreases gradually in intensity without any sign of discontinuity until it merges at high velocities with the bright EHV component. This EHV component appears in the spectrum as a separate secondary peak of intensity $0.5-1 \mathrm{~K}$ and centered around $V_{\mathrm{LSR}}=70 \mathrm{~km} \mathrm{~s}^{-1}$ in L1448 and $-35 \mathrm{~km} \mathrm{~s}^{-1}$ in I04166. In L1448, the EHV component is detected just above the noise level in the ${ }^{13} \mathrm{CO}(2-1)$ spectrum, while in I04166, the EHV feature can only be seen in the main isotopologues.

To carry out our abundance analysis, we need to define velocity limits for the wing and EHV components of each outflow, and we use for this the CO spectra in Fig. 2. For the EHV regime, we define its limits from the velocities at which this feature dominates the emission in the spectrum: $V_{\mathrm{LSR}}$ from 55 to $85 \mathrm{~km} \mathrm{~s}^{-1}$ in L1448 and -26 to $-43 \mathrm{~km} \mathrm{~s}^{-1}$ in I04166 (vertical dotted lines in Fig. 2). For the wing regime, we take as high velocity limit the lower value of the EHV component, as there is no clear gap between the two emissions in the spectra. Selecting the low velocity limit for the wing regime requires an additional consideration, as the wing emission merges smoothly with the optically thick ambient component. To guarantee that our wing contribution is optically thin in $\mathrm{CO}$, we have calculated the ratio between the $\mathrm{CO}(2-1)$ and ${ }^{13} \mathrm{CO}(2-1)$ spectra and determined the velocity at which the ratio becomes higher than 50 . This ratio is approximately equal to the expected ${ }^{12} \mathrm{C} /{ }^{13} \mathrm{C}$ abundance ratio in the local ISM ( $\approx 57$, Langer \& Penzias 1990), and is comparable to the maximum value measured with our data due to the limited $S / N$ ratio of the ${ }^{13} \mathrm{CO}(2-1)$ spectrum. According to our estimate, it is reached at $V_{\mathrm{LSR}}=10 \mathrm{~km} \mathrm{~s}^{-1}$ in L1448 and at $4 \mathrm{~km} \mathrm{~s}^{-1}$ in I04166, which from now on will be used as lower velocity limits for the (optically thin) wing regime.

Although the above velocity limits help separate the outflow into its two main components, velocity is not the only property that defines the wing or the EHV regime. Interferometer maps of the $\mathrm{CO}$ emission show that each regime corresponds to a different part of the outflow, with the wing emission arising from a shell and the EHV component lying along a jet (e.g. Santiago-García et al. 2009). These two components may therefore appear to overlap in velocity towards certain directions, but this does not necessarily imply that they spatially coexist in the outflow. Indeed, we will see below that the wing component seems to continue to higher velocities inside the EHV regime. Such an extension is very weak, and can be usually ignored when estimating the EHV contribution. It can however be detected in species with weak EHV emission, like HCN and possibly CS, and in those cases, it needs to be handled with some care.

\subsection{Overview of the molecular survey}

Figure 3 shows the spectra of the different high density tracers detected towards the L1448 and I04166 outflow target positions. As can be seen, a number of molecules have detectable emission in one or both outflow regimes, and overall, the spectra of most species resemble the $\mathrm{CO}$ spectra discussed in the previous section. Bright emission in the wing regime is seen, among others, in the lines of $\mathrm{HCN}, \mathrm{SO}$, and in the low $J$ transitions of SiO, while weaker but still noticeable wing emission can be seen in the spectra of $\mathrm{CH}_{3} \mathrm{OH}, \mathrm{CS}$, and $\mathrm{HCO}^{+}$.

As Fig. 3 also shows, several species present bright emission in the EHV regime. The SiO lines display the brightest EHV features, and have peak intensities of $\sim 100 \mathrm{mK}$. EHV SiO emission has previously been detected both in L1448 and I04166, 

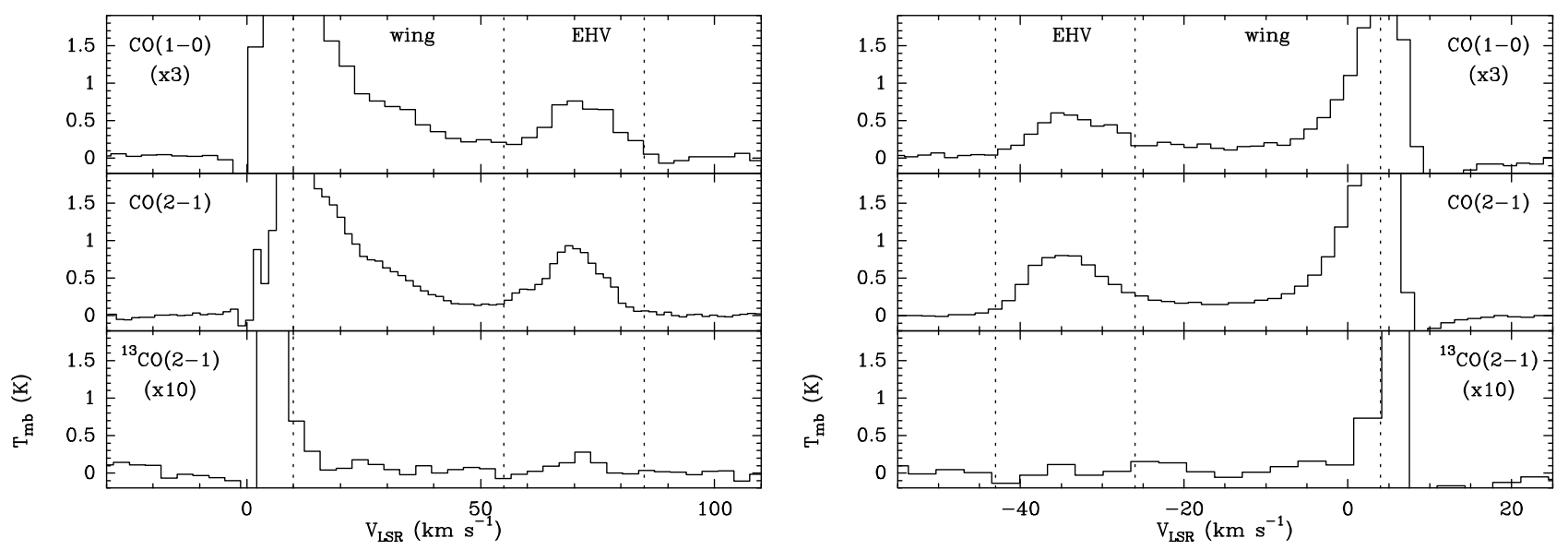

Fig. 2. $\mathrm{CO}(J=1-0), \mathrm{CO}(2-1)$, and ${ }^{13} \mathrm{CO}(2-1)$ spectra towards the target positions of the molecular survey in the L1448 (left) and I04166 (right) outflows. The intensity scale of the spectra has been truncated to better show the two velocity components of the outflow, the "wing" and the EHV regime, whose limits are indicated by vertical dotted lines. Negative features near the ambient velocity of each source $\left(V_{\mathrm{LSR}}=5.0 \mathrm{~km} \mathrm{~s}{ }^{-1}\right.$ in L1448 and $6.7 \mathrm{~km} \mathrm{~s}^{-1}$ in I04166) are artifacts of the wobbler switch observing mode, and do not affect the outflow emission studied here.

and this molecule has been so far the only known tracer of the EHV gas apart from CO (Bachiller et al. 1991; Nisini et al. 2007; Santiago-García et al. 2009). As our observations indicate, $\mathrm{SiO}$ is not the only dense gas tracer detectable in this outflow component. The SO molecule, for example, also presents significant EHV emission in both L1448 and I04166. EHV components were detected in $\mathrm{SO}\left(J_{\mathrm{N}}=3_{2}-2_{1}\right)$ and $\mathrm{SO}\left(J_{\mathrm{N}}=4_{3}-3_{2}\right)$ with an intensity of $\approx 50 \mathrm{mK}$, or about half of that of $\mathrm{SiO}$, and in the brighter L1448 outflow, the higher excitation $\operatorname{SO}\left(J_{\mathrm{N}}=\right.$ $6_{5}-5_{4}$ ) transition was also detected at a $4 \sigma$ level (not shown). Additional species with definitive detection of EHV emission are $\mathrm{CH}_{3} \mathrm{OH}$ (L1448 and likely I04166) and $\mathrm{H}_{2} \mathrm{CO}$ (L1448 only). They present intensities on the order of $10 \mathrm{mK}$, and their discussion is deferred to Sect. 3.4, where a number of weak detections and upper limits for the EHV gas will be further studied.

Before starting our analysis of the outflow chemical composition, we note that two main results from our survey can already be inferred from the direct inspection of the spectra in Fig. 3. The first result is that despite their factor of 10 difference in energetics, the L1448 and I04166 outflows present very similar excitation and chemical composition in both the wing and the EHV components. This can be noticed from the similar trends seen in Fig. 3 towards the two outflows. For example, in $\mathrm{SiO}$, both outflows present an EHV emission with all transitions up to $J=5-4$ having similar intensities $(\approx 100 \mathrm{mK})$ while, again in both outflows, the wing emission fades quickly in the high$\mathrm{J}$ lines. Also, both outflows present a combination of relatively weak $\mathrm{CS}$ and $\mathrm{HCO}^{+}$wings and very prominent $\mathrm{HCN}$ wing. This all suggests that the pattern of abundances in the two objects is very similar.

A second result that can be derived from an inspection of the line profiles is that the wing and the EHV components differ significantly in their chemical composition. This can be inferred from a lack of correlation between the intensity of the wing emission and the detection or not of an EHV feature. An extreme example of this behavior is the $\mathrm{HCN}(1-0)$ line, which has stronger wing emission than transitions of similar EinsteinA coefficient, like those of $\mathrm{SiO}$ and $\mathrm{SO}$, but that has no detectable EHV secondary feature in the spectrum (the HCN emission in this range seems only the continuation of the wing, see Sect. 3.4). Such a different behavior of the two outflow components is a first indication that they have undergone different chemical processing, which is a hint of a possible different physical origin of the wing and the EHV gas. Before further discussion, however, we need to investigate the internal chemical structure of the wing component.

\subsection{Two chemical regimes in the outflow wing component}

The outflow wing appears in the CO spectra as a single component with no internal structure, but in species like $\mathrm{SiO}, \mathrm{CS}$, or $\mathrm{H}_{2} \mathrm{CO}$, the wing presents a more complex shape. To illustrate this behavior, we present in Fig. 4 spectra for a few representative species together with scaled-down versions of the CO line profile. As can be seen, especially in the higher $S$ / $N$ L1448 data, the lines present different wing shapes, and only the SO spectrum resembles the $\mathrm{CO}$ profile over the whole wing regime. The $\mathrm{CS}$ and $\mathrm{SiO}$ profiles, present two different wing sections: a low velocity part in which the intensity lies below the (scaled-down) CO spectrum and a high velocity part in which the intensity follows the CO line shape (a vertical dashed line has been drawn to separate these two wing sections). In contrast with $\mathrm{SO}$ and $\mathrm{SiO}$, $\mathrm{H}_{2} \mathrm{CO}$ presents a different (and unique) behavior. Its line profile seems to match the $\mathrm{CO}$ spectrum in the low velocity section of the wing while it drops below $\mathrm{CO}$ at higher velocities. As Fig. 4 shows, despite the variety of line profiles and wing sections, the behavior of each species is remarkably similar in both outflows. This suggests that the line changes we see in the figure are more related to the peculiar chemistry of each species than to the details of the outflow.

Another remarkable property of the line profiles in Fig. 4 is the transition between the two wing sections. For a given outflow, this transition occurs at the same velocity for all species, and often in a relatively sudden way. In L1448, both CS and $\mathrm{SiO}$ spectra have $\mathrm{a} \approx 5 \mathrm{~km} \mathrm{~s}^{-1}$-wide velocity section just below $V_{\mathrm{LSR}}=20 \mathrm{~km} \mathrm{~s}^{-1}$ where the wing shape reverses, and the intensity of the emission increases slightly with velocity before reaching the faster wing section where it matches the $\mathrm{CO}$ profile. It is unlikely that this dip in the wing emission arises from self-absorption by ambient gas because the dip is red-shifted by $10 \mathrm{~km} \mathrm{~s}^{-1}$ with respect to the ambient velocity. The dip is also unlikely to arise from contamination by the reference position because it was seen under different observing conditions and in different epochs. It must represent a real feature of the emission. 
L1448

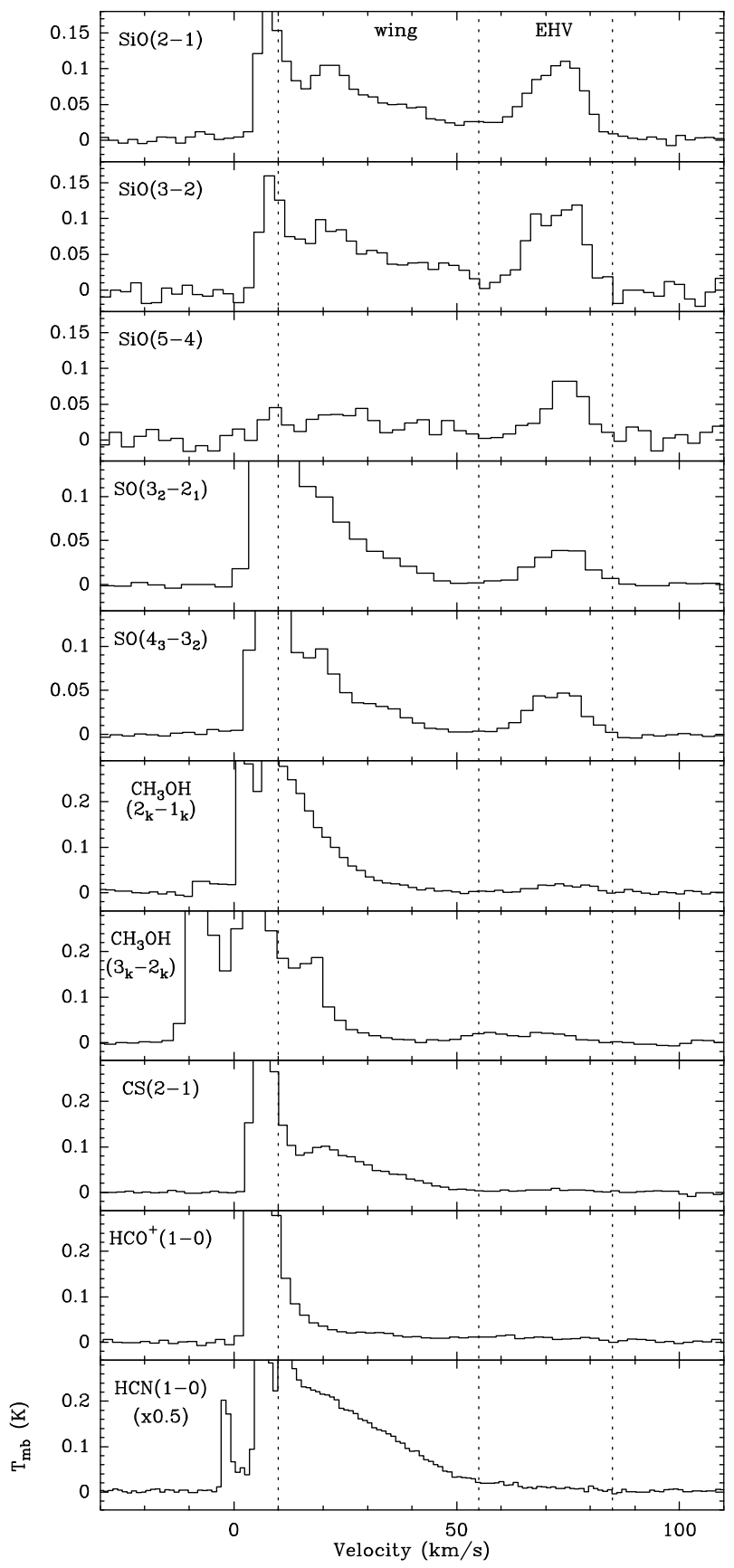

I04166

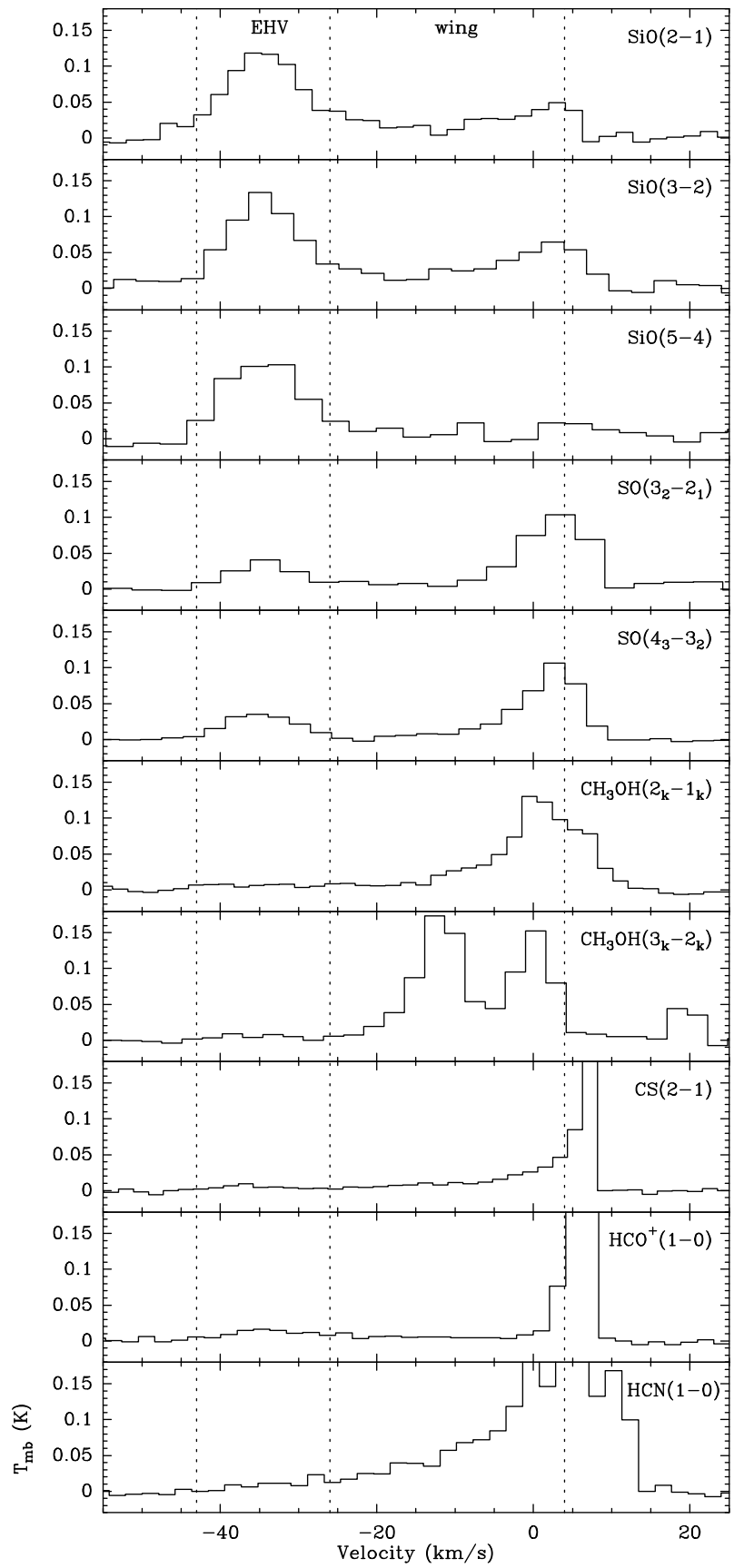

Fig. 3. Spectra from the high density tracers with detected outflow emission in the L1448 (left) and I04166 (right) survey targets. As in Fig. 2, the vertical dotted lines mark the boundaries of the wing and EHV outflow regimes. Note the prominent EHV features in the SiO and SO lines, and the prominent wings of lines from other species like HCN. Note also how the outflow regimes partly overlap in the multicomponent spectra of $\mathrm{CH}_{3} \mathrm{OH}$. The analysis used to disentangle these components is described in Appendix C.

In I04166, no sharp transition is seen between the two wing sections, but the $\mathrm{CS}$ and $\mathrm{SiO}$ spectra seem to match the fast part of the $\mathrm{CO}$ wing at the same velocity near $V_{\mathrm{LSR}}=-7 \mathrm{~km} \mathrm{~s}^{-1}$.

The above differences in the shape of the profiles indicate that for most molecules, the ratio of intensity with respect to $\mathrm{CO}$ changes with velocity over the wing regime. Barring excitation and optical depth effects (discussed in the following sections), such a change in the intensity ratio must correspond to a change in the ratio of the abundance with respect to $\mathrm{CO}$. The wing regime, therefore, seems not made of gas having uniform composition, but to consist of at least two regimes with different chemical properties. To include this behavior in our abundance analysis, from now on we will consider the two wing regimes separately, and we will refer to them as the slow wing or s-wing and the fast wing or f-wing. Their velocity limits, and those of the EHV component, are indicated by vertical dotted lines in Fig. 4 and summarized in Table 2.

\subsection{Molecular emission in the EHV regime}

The emission of molecules in the EHV regime deserves special attention. Before our survey was carried out, only $\mathrm{CO}$ and $\mathrm{SiO}$ had been detected in this outflow component, but as shown in Sect. 3.2, SO also presents bright EHV components 

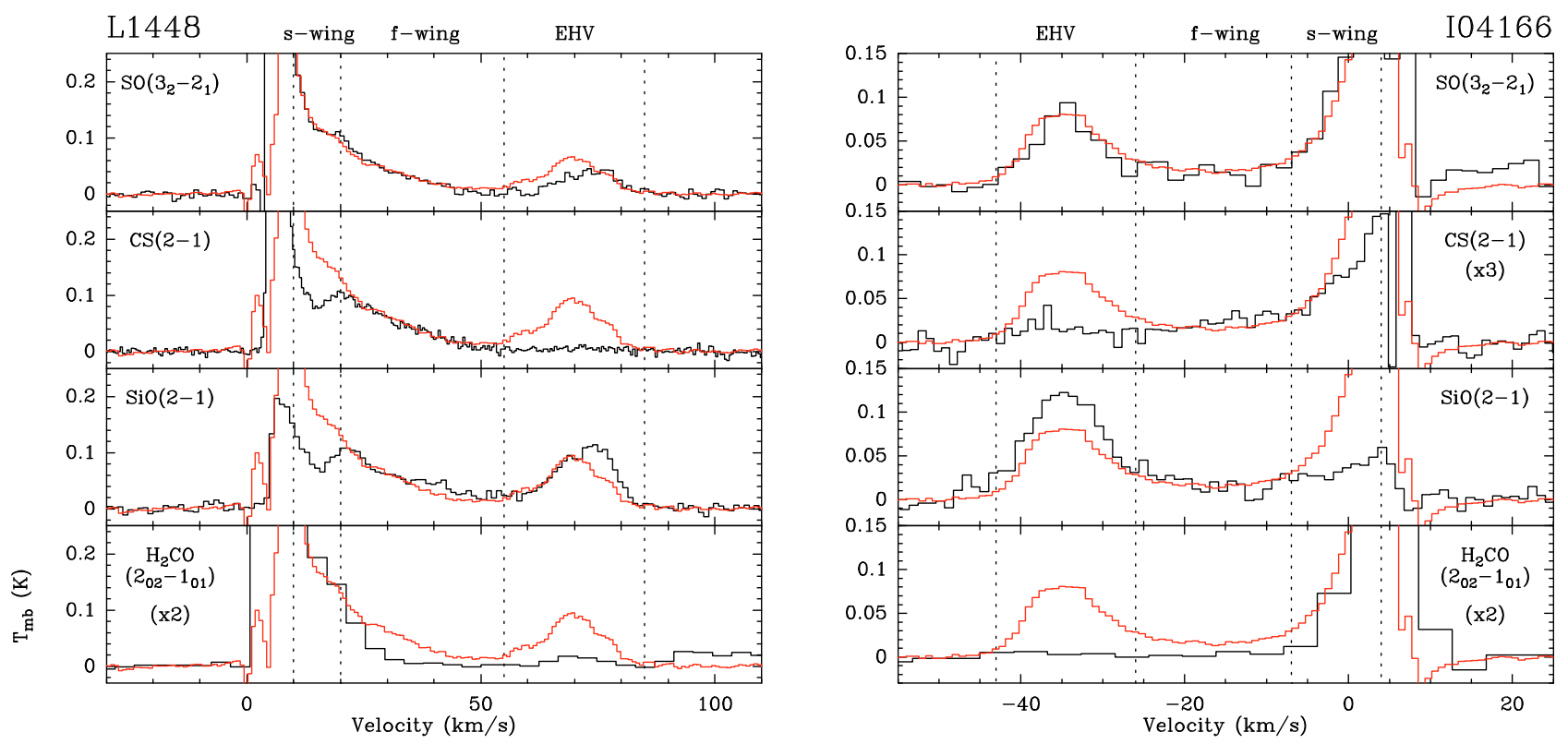

Fig. 4. Comparison of spectra from selected dense gas tracers (black) with scaled-down versions of the $\operatorname{CO}(2-1)$ spectrum (red) to illustrate the existence of two different chemical components in the wing regime of the L1448 and I04166 outflows. Note how in the SO, CS, and SiO spectra, the fast wing (f-wing) component matches well the CO spectrum, while the slow wing (s-wing) component follows a different trend: in SO, it matches the $\mathrm{CO}$ spectrum, while in $\mathrm{CS}$ and $\mathrm{SiO}$, it lies below it. In contrast, the $\mathrm{H}_{2} \mathrm{CO}$ wing matches the $\mathrm{CO}$ s-wing but lies below the f-wing at higher velocities. This different behavior of the spectra in the s-wing and f-wing regimes suggests that the composition of these two parts of the outflow follow different trends and need to be studied separately.

Table 2. Velocity ranges of the slow wing (s-wing), fast wing (f-wing), and extremely high velocity (EHV) regimes.

\begin{tabular}{lccc}
\hline \hline Target position & $\begin{array}{c}\text { s-wing } \\
\left(\mathrm{km} \mathrm{s}^{-1}\right)\end{array}$ & $\begin{array}{c}\text { f-wing } \\
\left(\mathrm{km} \mathrm{s}^{-1}\right)\end{array}$ & $\begin{array}{c}\text { EHV } \\
\left(\mathrm{km} \mathrm{s}^{-1}\right)\end{array}$ \\
\hline L1448 $\left(16^{\prime \prime},-34^{\prime \prime}\right)^{1}$ & {$[10,20]$} & {$[20,55]$} & {$[55,85]$} \\
$\mathrm{I} 04166\left(8^{\prime \prime}, 14^{\prime \prime}\right)^{2}$ & {$[-7,4]$} & {$[-26,-7]$} & {$[-43,-26]$} \\
\hline
\end{tabular}

Notes. ${ }^{(1)}$ Offsets with respect to $\alpha(J 2000)=3^{\mathrm{h}} 25^{\mathrm{m}} 38^{\mathrm{s}} .9, \delta(J 2000)=$ $+30^{\circ} 44^{\prime} 05^{\prime \prime}$; ${ }^{(2)}$ offsets with respect $\alpha(J 2000)=4^{\mathrm{h}} 19^{\mathrm{m}} 42^{\mathrm{s}} .5, \delta(J 2000)=$ $+27^{\circ} 13^{\prime} 36^{\prime \prime}$.

in at least two transitions towards both L1448 and I04166. The SO molecule is therefore a relatively bright tracer of this fastest outflow component, and in Appendix A we present the results of a small SO survey along the axes of the L1448 and I04166 outflows illustrating the widespread nature of the EHV SO emission.

In addition to $\mathrm{CO}, \mathrm{SiO}$, and $\mathrm{SO}$, our observations revealed weak $(\sim 10 \mathrm{mK})$ high-velocity emission in a number of additional species, some of them presenting a distinct EHV feature while others of a less clear nature. This is illustrated in Fig. 5, which shows an expanded view of the spectra for all species with detectable emission at high velocities, together with scaleddown versions of the $\operatorname{SO}\left(J_{\mathrm{N}}=3_{2}-2_{1}\right)$ line to indicate the expected position of the EHV regime. (For the $\mathrm{CH}_{3} \mathrm{OH}$ and $\mathrm{HCN}$ lines, the SO models are in fact templates of the multi component spectrum and were created using the method described in Appendix C.) Given the weak emission of the features, the variety of line shapes, and the possible contamination from lines of additional molecules, we will consider each line separately.

As Fig. 5 shows, both the $\mathrm{CH}_{3} \mathrm{OH}\left(2_{k}-1_{k}\right)$ and $\mathrm{CH}_{3} \mathrm{OH}\left(3_{k}-2_{k}\right)$ spectra towards the L1448 outflow present distinct secondary peaks in the velocity range expected for the EHV component $\left(V_{\mathrm{LSR}}=55-85 \mathrm{~km} \mathrm{~s}^{-1}\right)$. These peaks match closely the EHV features predicted by the SO template, indicated in the figure by a red line plotted below the $\mathrm{CH}_{3} \mathrm{OH}$ spectrum. For $\mathrm{CH}_{3} \mathrm{OH}\left(3_{k}-2_{k}\right)$, the $\mathrm{SO}$ template predicts a significantly broader EHV component due to the overlap of the contributions from the $\mathrm{E}$ and $\mathrm{A} \mathrm{CH}_{3} \mathrm{OH}$ species, and indeed the observed $\mathrm{CH}_{3} \mathrm{OH}\left(3_{k}-2_{k}\right)$ EHV feature is significantly broader than in $\mathrm{CH}_{3} \mathrm{OH}\left(2_{k}-1_{k}\right)$. We consider this double match between observations and $\mathrm{SO}$ templates a definitive detection of $\mathrm{CH}_{3} \mathrm{OH}$ in the EHV gas of the L1448 outflow.

The situation in I04166 is harder to evaluate due to a significantly (factor of 2) weaker $\mathrm{CH}_{3} \mathrm{OH}$ signal and to the expected overlap in $\mathrm{CH}_{3} \mathrm{OH}\left(3_{k}-2_{k}\right)$ between the EHV feature and a group of weak (mostly $k=2$ ) $\mathrm{CH}_{3} \mathrm{OH}$ ambient-velocity components whose position is indicated in the figure by a horizontal bar. As Fig. 5 shows, both $\mathrm{CH}_{3} \mathrm{OH}$ spectra present significant emission at the expected velocity of the EHV gas, but the lack of a distinct secondary peak isolated from the rest of the outflow wing makes this detection less clear than that in the L1448 outflow.

Another convincing detection of an EHV feature can be seen in the $\mathrm{H}_{2} \mathrm{CO}$ spectrum towards the L1448 outflow. Figure 5 shows that the $\mathrm{H}_{2} \mathrm{CO}\left(2_{02}-1_{01}\right)$ line presents a well-defined secondary peak at the expected velocity for the EHV gas as indicated by the $\mathrm{SO}$ profile, again shown in red below the $\mathrm{H}_{2} \mathrm{CO}$ spectrum. This $\mathrm{H}_{2} \mathrm{CO}$ feature stands out in the spectrum as a distinct component, and is well separated from the brighter peak near $V_{\mathrm{LSR}}>100 \mathrm{~km} \mathrm{~s}^{-1}$ that corresponds to the ambient and outflow wing contributions from $\mathrm{HC}_{3} \mathrm{~N}(16-15)$. We thus consider this feature as a clear detection of $\mathrm{H}_{2} \mathrm{CO}$ in the EHV component of the L1448 outflow.

As with $\mathrm{CH}_{3} \mathrm{OH}$, no clear $\mathrm{H}_{2} \mathrm{CO}$ EHV feature can be seen in the weaker $\mathrm{I} 04166$ spectrum. Our data only provides an upper limit to the $\mathrm{H}_{2} \mathrm{CO}$ emission in this outflow component, and a deeper integration is needed to test whether there is any difference between the presence of $\mathrm{H}_{2} \mathrm{CO}$ in the EHV component of the two outflows. 

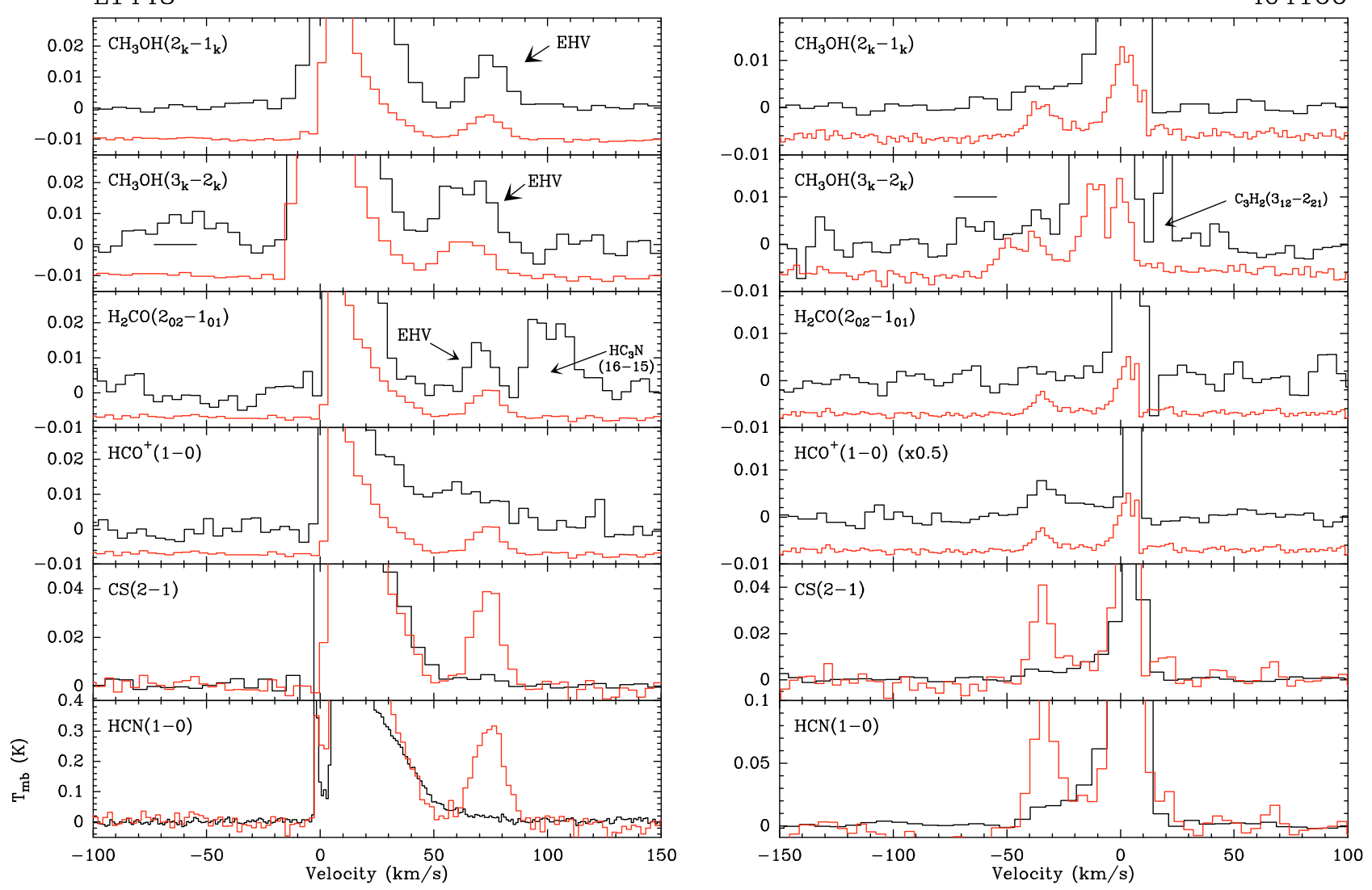

Fig. 5. Evidence for EHV emission in $\mathrm{CH}_{3} \mathrm{OH}$ and $\mathrm{H}_{2} \mathrm{CO}$ towards the $\mathrm{L} 1448$ outflow, together with possible detections in $\mathrm{HCO}^{+}$, CS, and $\mathrm{HCN}$. The black histograms represent the data and the red histograms are scaled-down $\mathrm{SO}\left(3_{2}-2_{1}\right)$ spectra to illustrate the expected location of the EHV peak (near $V_{\mathrm{LSR}}=70 \mathrm{~km} \mathrm{~s}^{-1}$ in L1448 and near $-35 \mathrm{~km} \mathrm{~s}^{-1}$ in I04166). For $\mathrm{CH}_{3} \mathrm{OH}$ and $\mathrm{HCN}$, the red histogram is a synthetic spectrum derived using the $\mathrm{SO}\left(3_{2}-2_{1}\right)$ line as a template (see Appendix $\mathrm{C}$ for further details). Note the weak EHV peaks and the gradual transition from well defined secondary components in $\mathrm{CH}_{3} \mathrm{OH}$ and $\mathrm{H}_{2} \mathrm{CO}$ to more wing-like features in $\mathrm{HCO}^{+}, \mathrm{CS}$, and $\mathrm{HCN}$. Several unrelated lines are indicated, and the horizontal lines in the $\mathrm{CH}_{3} \mathrm{OH}\left(3_{k}-2_{k}\right)$ spectra mark the position of weak $k=2$ components that may contaminate the EHV feature in I04166. See text for a discussion of the reliability of each detection. SO was chosen as a template due to its similar behavior to CO and higher sensitivity to dense gas.

The third species with a likely EHV feature in the spectrum is $\mathrm{HCO}^{+}$. As Fig. 5 shows, the $\mathrm{HCO}^{+}(1-0)$ line from the $\mathrm{L} 1448$ outflow presents an almost continuous wing with a hint of a relative maximum near the EHV velocity range. In the I04166 spectrum, on the other hand, $\mathrm{HCO}^{+}(1-0)$ presents a distinct emission peak at the velocity expected for the EHV regime, suggesting that at least part of the $\mathrm{HCO}^{+}$emission at high velocities truly arises from the EHV gas. These two $\mathrm{HCO}^{+}$spectra illustrate a trend, further seen in $\mathrm{CS}$ and $\mathrm{HCN}$, of increasing confusion between the EHV feature and what seems to be a high-velocity continuation of the wing emission. As mentioned in Sect. 3.1, the wing emission in an outflow probably does not end at the velocity at which the EHV component starts to dominate the spectrum, but it continues to higher velocities overlapping with the EHV gas. In the lines of $\mathrm{CO}$ (also $\mathrm{SiO}$ and $\mathrm{SO}$ ), the EHV emission is brighter than the wing, so it dominates the spectrum. In $\mathrm{HCO}^{+}$, CS, and $\mathrm{HCN}$, the weak EHV emission barely (if at all) stands out over the wing emission, and its separation from the fastest wing is ambiguous.

The last two species that we consider are CS and HCN. Their spectra present statistically significant emission at velocities of the EHV regime (bottom two panels in Fig. 5), but the nature of this emission is unclear. The CS(2-1) spectrum in both the L1448 and I04166 outflows presents rather flat emission in the expected range of the EHV component, with a possible hint of a secondary peak in the L1448 outflow. The I04166 spectrum may be slightly contaminated by a pair of $\mathrm{C}_{3} \mathrm{H}$ lines, so its shape should not be used to judge a possible EHV peak. The $\mathrm{HCN}(1-0)$ spectrum, on the other hand, presents smooth winglike emission at the velocities expected for the EHV component (as predicted by the SO template), suggesting that in this molecule the wing emission overwhelms any possible contribution from the EHV gas. Thus, while it is possible that the EHV component emits non-negligibly in CS(2-1), we consider that most of the high velocity $\mathrm{HCN}(1-0)$ emission arises from the extended wing component and not from the EHV gas.

In summary, our observations show definite emission from the EHV component in $\mathrm{CH}_{3} \mathrm{OH}$ and $\mathrm{H}_{2} \mathrm{CO}$ towards the $\mathrm{L} 1448$ outflow, likely emission in $\mathrm{HCO}^{+}$for both outflows, possible emission in CS, and unlikely emission in HCN. The results towards I04166 are compatible with those towards L1448. Given the several hours of integration needed to obtain the spectra of Fig. 5, it seems unlikely that a more definitive conclusion for the marginal cases can be reached in the foreseeable future.

\subsection{Column density estimates}

We start our abundance analysis by estimating the column density of each species in our survey. As a first step, we integrate the observed intensity inside each of the three velocity ranges into 
which we have divided the outflow emission (s-wing, f-wing, and EHV, see Table 2). For species like CO, SiO, SO, CS, and $\mathrm{CH}_{3} \mathrm{OH}$, we have observed more than one transition, so we can use a population diagram analysis to check whether the molecular energy levels are in LTE, and if so, to derive an excitation temperature and a total column density (Goldsmith \& Langer 1999). Throughout this analysis, we assume that the outflow emission is optically thin. For the $\mathrm{CO}$ lines the condition is guaranteed by the comparison with the ${ }^{13} \mathrm{CO}$ data (Sect. 3.1), and for other species, the data already provides a strong hint of the emission being thin because the observed intensities are on the order of $0.1 \mathrm{~K}$, while the excitation temperatures are close to $7 \mathrm{~K}$ (see below).

Before converting the observed line intensities into column densities, a number of corrections are needed. A first correction is required to compensate for the fact that transitions with different frequencies were observed using different beam sizes. As interferometer maps show (e.g., Dutrey et al. 1997; Santiago-García et al. 2009), the outflow emission is generally compact, especially in the EHV regime, so the smaller beam size of the high frequency transitions is expected to enhance their emission. As these lines usually correspond to high excitation levels, this effect can introduce a systematic bias in the column density estimate, and needs to be corrected. For this, we have used high-resolution $\mathrm{CO}$ data to model the dependence of the observed intensity with beam size in each of the outflow regimes, and we have derived correction factors to convert the emission of each transition into the expected value for a $16^{\prime \prime}$ beam (the approximate mean value of our beam sizes). The good match between line shapes of the high density tracers and CO (Fig. 4) suggests that these CO-based correction factors can be applied to the different species in our survey. A full description of the procedure is presented in Appendix B.

A second correction is required to disentangle the overlap between multiple lines in the spectra of $\mathrm{HCN}$ and $\mathrm{CH}_{3} \mathrm{OH}$. In these spectra, the ambient lines are usually well separated, but the outflow regimes are not. Fortunately, the overlap between outflow components is only partial, and the outflow contribution to each individual line seems to always produce the same type of profile. Thus, we have modeled each multi-component spectrum as consisting of partly overlapping lines all having the same spectral shape and each centered at the velocity given by their rest frequency. As templates for these components, we have used observed lines, usually from $\mathrm{SO}$ and $\mathrm{SiO}$, and we have derived their relative intensities from a fit to the full spectrum (see Appendix $\mathrm{C}$ for further details).

A final consideration in the column density analysis is needed because the excitation temperatures derived for the outflow gas are generally low due to subthermal excitation $(\approx 7 \mathrm{~K})$, so that the common assumption that $T_{\mathrm{ex}}$ is much higher than $T_{\mathrm{bg}}$, the cosmic background temperature, is not guaranteed. In this situation (and assuming that the line is thin), the column density of the upper level of one transition $\left(N_{\mathrm{u}}\right)$ is related to the observed integrated intensity $W$ by

$N_{\mathrm{u}}=\frac{8 \pi k v^{2} W}{h c^{3} A_{\mathrm{ul}}}\left[1-\frac{\mathrm{e}^{h v / k T_{\text {ex }}}-1}{\mathrm{e}^{h v / k T_{\text {bg }}}-1}\right]^{-1}$,

where $k$ is Boltzmann's constant, $v$ the line frequency, $h$ Planck's constant, $c$ the speed of light, and $A_{\mathrm{ul}}$ the Einstein $A$-coefficient. The term in square brackets, ignored in the standard (hightemperature) population diagram analysis, makes the column density estimate depend on $T_{\text {ex }}$, which is not known until the analysis has been performed. Fortunately, this term is usually close enough to 1 that $N_{\mathrm{u}}$ can be solved iteratively. In our computation, we first assume that the term is equal to 1 and derive $T_{\text {ex }}$ using the standard population analysis. Then, we iterate the LTE estimate recalculating $N_{\mathrm{u}}$ with the $T_{\mathrm{ex}}$ value from the previous iteration. Convergence to a level of $10^{-4}$ in $T_{\mathrm{ex}}$ is usually reached in 4-5 iterations.

As a result of our LTE analysis, we have derived $T_{\text {ex }}$ and column density $\left(N_{\text {TOT }}\right)$ values for each outflow component in all species with more than one observed transition. These values are presented Table 3, and population diagrams for $\mathrm{CO}, \mathrm{SO}$, and $\mathrm{SiO}$ are shown in Fig. 6 for illustration. As the table and figure show, the $T_{\text {ex }}$ values for $\mathrm{CO}$ are close to $15-20 \mathrm{~K}$ in both outflows, while the $T_{\text {ex }}$ values for the high-density tracers are lower than $10 \mathrm{~K}$ and usually close to $7-8 \mathrm{~K}$, also in both outflows and in all the velocity regimes. This difference in $T_{\mathrm{ex}}$ between $\mathrm{CO}$ and the other tracers indicates that the emission we observe in all the outflow regimes arises from gas where the high-density tracers are subthermally excited while $\mathrm{CO}$ is probably thermalized (if $\mathrm{CO}$ were subthermal, then $T_{\mathrm{ex}}$ for the high density tracers would be close to the cosmic background temperature). Such excitation conditions are satisfied if the emitting gas has a kinetic temperature of around $20 \mathrm{~K}$ and a density close to $10^{6} \mathrm{~cm}^{-3}$, conditions which match closely those predicted by realistic models of post-shock gas (Bergin et al. 1998). A more detailed analysis of the excitation conditions in the outflow gas for a larger sample of objects will be presented in Santiago-García et al. (2010, in preparation).

The similar $T_{\text {ex }}$ values shown in Table 3 for the high-density tracers in the outflow suggest that for those species for which only one transition was observed in our survey (e.g., $\mathrm{HCO}^{+}$, $\mathrm{H}_{2} \mathrm{CO}, \mathrm{HCN}$ ), we can safely assume a single excitation value in the column density calculation. Based on the $\mathrm{SiO}, \mathrm{SO}$, and $\mathrm{CS}$ estimates, we take as default value $T_{\mathrm{ex}}=7 \pm 1 \mathrm{~K}$, and use it to derive column densities for the species with only one observed transition. For species with no detection, we use the spectrum noise level together with the default $T_{\mathrm{ex}}$ value to derive upper limits to their column density. Table 3 summarizes all the results.

\section{Analysis of the outflow abundances}

\subsection{The similar composition of the $L 1448$ and 104166 outflows}

As Table 3 shows, the CO column densities in the L1448 outflow are significantly higher than in I04166. The ratio between the two is 1.5 in the s-wing regime, 4.9 in the f-wing regime, and 1.8 in the EHV regime, and these numbers are in general agreement with the fact that the L1448 outflow is more massive and energetic than that of I04166. To compensate for this difference, and to properly compare the abundance of the different molecular species in the two sources, we normalize each column density by the $\mathrm{CO}$ value in the same regime, working from now on with abundances with respect to CO. Such CO-normalized abundances (referred to hereafter simply as "abundances" unless needed to avoid ambiguity) are expected to be proportional to the true molecular abundances (i.e., with respect to $\mathrm{H}_{2}$ ) given the stability of $\mathrm{CO}$ under a number of shock conditions (e.g., Bergin et al. 1998). Changes in the nature of the chemistry, however, like from shock-processed to protostellar-wind origin, can potentially affect the $\mathrm{CO}$ abundance and therefore the normalization factor (see Glassgold et al. 1991).

In Table 4 we present abundance estimates with respect to $\mathrm{CO}$ for all the molecules in our survey, as derived using the values given in Table 3. As can be seen, the highest CO-normalized 
M. Tafalla et al.: A molecular survey of outflow gas

Table 3. Molecular column densities and excitation temperatures derived towards the L1448 and I04166 outflow targets.

\begin{tabular}{|c|c|c|c|c|c|c|}
\hline \multirow[b]{3}{*}{ MOLEC } & \multicolumn{6}{|c|}{ L1448 } \\
\hline & \multicolumn{2}{|l|}{ s-wing } & \multicolumn{2}{|l|}{ f-wing } & \multicolumn{2}{|l|}{ EHV } \\
\hline & $N_{T}\left(\mathrm{~cm}^{-2}\right)$ & $T_{\mathrm{ex}}(\mathrm{K})$ & $N_{T}\left(\mathrm{~cm}^{-2}\right)$ & $T_{\mathrm{ex}}(\mathrm{K})$ & $N_{T}\left(\mathrm{~cm}^{-2}\right)$ & $T_{\mathrm{ex}}(\mathrm{K})$ \\
\hline $\mathrm{CO}$ & $1.0 \times 10^{16} \pm 1 \times 10^{14}$ & $18.6 \pm 0.3$ & $7.4 \times 10^{15} \pm 2 \times 10^{14}$ & $15.6 \pm 0.5$ & $5.7 \times 10^{15} \pm 2 \times 10^{14}$ & $11.1 \pm 0.3$ \\
\hline $\mathrm{SiO}$ & $2.4 \times 10^{12} \pm 2 \times 10^{11}$ & $5.6 \pm 0.3$ & $4.7 \times 10^{12} \pm 2 \times 10^{11}$ & $7.1 \pm 0.3$ & $6.4 \times 10^{12} \pm 2 \times 10^{11}$ & $7.0 \pm 0.3$ \\
\hline SO & $1.8 \times 10^{13} \pm 6 \times 10^{11}$ & $7.2 \pm 0.1$ & $1.4 \times 10^{13} \pm 9 \times 10^{11}$ & $7.9 \pm 0.3$ & $1.1 \times 10^{13} \pm 1 \times 10^{12}$ & $8.3 \pm 0.5$ \\
\hline $\mathrm{CS}$ & $5.5 \times 10^{12} \pm 2 \times 10^{11}$ & $6.3 \pm 0.1$ & $8.5 \times 10^{12} \pm 3 \times 10^{11}$ & $6.1 \pm 0.2$ & $7 \times 10^{11} \pm 3 \times 10^{11}$ & $7 \pm 1^{1}$ \\
\hline $\mathrm{CH}_{3} \mathrm{OH}^{2}$ & $7.9 \times 10^{13} \pm 1.1 \times 10^{13}$ & $10 \pm 1$ & $5.1 \times 10^{13} \pm 1.5 \times 10^{13}$ & $11 \pm 4$ & $1.6 \times 10^{13} \pm 5 \times 10^{12}$ & $10 \pm 3$ \\
\hline $\mathrm{HC}_{3} \mathrm{~N}$ & $2.0 \times 10^{12} \pm 5 \times 10^{11}$ & $14 \pm 2$ & $2.4 \times 10^{12} \pm 1.4 \times 10^{12}$ & $11 \pm 3$ & $<1 \times 10^{12}$ & $7 \pm 1^{1}$ \\
\hline $\mathrm{HCO}^{+}$ & $9.3 \times 10^{11} \pm 2 \times 10^{10}$ & $7 \pm 1^{1}$ & $7.0 \times 10^{11} \pm 3 \times 10^{10}$ & $7 \pm 1^{1}$ & $5.0 \times 10^{11} \pm 4 \times 10^{10}$ & $7 \pm 1^{1}$ \\
\hline $\mathrm{p}-\mathrm{H}_{2} \mathrm{CO}$ & $1.9 \times 10^{12} \pm 3 \times 10^{11}$ & $7 \pm 1^{1}$ & $7.4 \times 10^{11} \pm 1 \times 10^{11}$ & $7 \pm 1^{1}$ & $2.3 \times 10^{11} \pm 3 \times 10^{10}$ & $7 \pm 1^{1}$ \\
\hline $\mathrm{HCN}$ & $9.7 \times 10^{12} \pm 1 \times 10^{12}$ & $7 \pm 1^{1}$ & $1.6 \times 10^{13} \pm 3 \times 10^{11}$ & $7 \pm 1^{1}$ & $1.2 \times 10^{12} \pm 2 \times 10^{11}$ & $7 \pm 1^{1}$ \\
\hline $\mathrm{SO}_{2}$ & $3.4 \times 10^{12} \pm 6 \times 10^{11}$ & $7 \pm 1^{1}$ & $<2 \times 10^{12}$ & $7 \pm 1^{1}$ & $<3 \times 10^{12}$ & $7 \pm 1^{1}$ \\
\hline $\mathrm{SiS}$ & $<5 \times 10^{11}$ & $7 \pm 1^{1}$ & $<2 \times 10^{12}$ & $7 \pm 1^{1}$ & $<1 \times 10^{12}$ & $7 \pm 1^{1}$ \\
\hline \multirow[t]{3}{*}{$\mathrm{H}_{2} \mathrm{~S}$} & $<3 \times 10^{12}$ & $7 \pm 1^{1}$ & $<5 \times 10^{12}$ & $7 \pm 1^{1}$ & $<4 \times 10^{12}$ & $7 \pm 1^{1}$ \\
\hline & \multicolumn{6}{|c|}{ I04166 } \\
\hline & \multicolumn{2}{|l|}{ s-wing } & \multicolumn{2}{|l|}{ f-wing } & \multicolumn{2}{|l|}{ EHV } \\
\hline MOLEC & $N_{T}\left(\mathrm{~cm}^{-2}\right)$ & $T_{\mathrm{ex}}(\mathrm{K})$ & $N_{T}\left(\mathrm{~cm}^{-2}\right)$ & $T_{\text {ex }}(\mathrm{K})$ & $N_{T}\left(\mathrm{~cm}^{-2}\right)$ & $T_{\mathrm{ex}}(\mathrm{K})$ \\
\hline $\mathrm{CO}$ & $6.5 \times 10^{15} \pm 1 \times 10^{14}$ & $24.5 \pm 0.6$ & $1.5 \times 10^{15} \pm 1 \times 10^{14}$ & $17 \pm 1$ & $3.2 \times 10^{15} \pm 1 \times 10^{14}$ & $18 \pm 1$ \\
\hline $\mathrm{SiO}$ & $1.2 \times 10^{12} \pm 2 \times 10^{11}$ & $7.3 \pm 0.7$ & $1.1 \times 10^{12} \pm 1 \times 10^{11}$ & $8.0 \pm 0.6$ & $4.0 \times 10^{12} \pm 1 \times 10^{11}$ & $9.6 \pm 0.1$ \\
\hline SO & $1.2 \times 10^{13} \pm 8 \times 10^{11}$ & $6.0 \pm 0.2$ & $3.6 \times 10^{12} \pm 2 \times 10^{12}$ & $5.0 \pm 0.8$ & $7.3 \times 10^{12} \pm 1 \times 10^{12}$ & $6.6 \pm 0.4$ \\
\hline $\mathrm{CS}$ & $2.1 \times 10^{12} \pm 1 \times 10^{11}$ & $5.6 \pm 0.2$ & $1.4 \times 10^{12} \pm 3 \times 10^{11}$ & $4.6 \pm 0.5$ & $5.4 \times 10^{11} \pm 6 \times 10^{10}$ & $7 \pm 1^{1}$ \\
\hline $\mathrm{CH}_{3} \mathrm{OH}^{2}$ & $5.2 \times 10^{13} \pm 8 \times 10^{12}$ & $11 \pm 1$ & $8.1 \times 10^{12} \pm 2 \times 10^{12}$ & $8 \pm 2$ & $6.3 \times 10^{12} \pm 1.8 \times 10^{12}$ & $7 \pm 2$ \\
\hline $\mathrm{HC}_{3} \mathrm{~N}$ & $<2 \times 10^{12}$ & $7 \pm 1^{1}$ & $<5 \times 10^{11}$ & $7 \pm 1^{1}$ & $<5 \times 10^{11}$ & $7 \pm 1^{1}$ \\
\hline $\mathrm{HCO}^{+}$ & $2.6 \times 10^{11} \pm 2 \times 10^{10}$ & $7 \pm 1^{1}$ & $1.9 \times 10^{11} \pm 3 \times 10^{10}$ & $7 \pm 1^{1}$ & $3.0 \times 10^{11} \pm 2 \times 10^{10}$ & $7 \pm 1^{1}$ \\
\hline $\mathrm{p}-\mathrm{H}_{2} \mathrm{CO}$ & $1.8 \times 10^{12} \pm 2 \times 10^{11}$ & $7 \pm 1^{1}$ & $<6 \times 10^{10}$ & $7 \pm 1^{1}$ & $<5 \times 10^{11}$ & $7 \pm 1^{1}$ \\
\hline $\mathrm{HCN}^{3}$ & $3.2 \times 10^{12} \pm 3 \times 10^{11}$ & $7 \pm 1^{1}$ & $3.4 \times 10^{12} \pm 3 \times 10^{11}$ & $7 \pm 1^{1}$ & $2.8 \times 10^{11} \pm 3 \times 10^{10}$ & $7 \pm 1^{1}$ \\
\hline $\mathrm{SO}_{2}$ & $<2 \times 10^{12}$ & $7 \pm 1^{1}$ & $<1 \times 10^{12}$ & $7 \pm 1^{1}$ & $<1 \times 10^{12}$ & $7 \pm 1^{1}$ \\
\hline $\mathrm{SiS}$ & $<5 \times 10^{11}$ & $7 \pm 1^{1}$ & $<1 \times 10^{12}$ & $7 \pm 1^{1}$ & $<7 \times 10^{11}$ & $7 \pm 1^{1}$ \\
\hline $\mathrm{H}_{2} \mathrm{~S}$ & $<5 \times 10^{12}$ & $7 \pm 1^{1}$ & $<2 \times 10^{13}$ & $7 \pm 1^{1}$ & $<3 \times 10^{12}$ & $7 \pm 1^{1}$ \\
\hline
\end{tabular}

Notes. ${ }^{(1)} T_{\mathrm{ex}}=7 \pm 1 \mathrm{~K}$ assumed; ${ }^{(2)}$ The $\mathrm{E}$ and A-forms of $\mathrm{CH}_{3} \mathrm{OH}$ were analyzed separately and the column density in the table is the sum of the two results ( $T_{\text {ex }}$ is the mean value). The $\mathrm{E} / \mathrm{A}$ ratio was found $\approx 1.2$ in both sources and for all outflow regimes; ${ }^{(3)} 10 \%$ uncertainty assumed for I04166 estimate of HCN column density.

Table 4. CO-normalized abundances $\left(\times 10^{4}\right)$ and enhancement factors with respect to dense core abundances ${ }^{1}$.

\begin{tabular}{|c|c|c|c|c|c|c|c|c|c|c|c|c|}
\hline \multirow[b]{2}{*}{ MOLEC } & \multicolumn{6}{|c|}{ L1448 } & \multicolumn{6}{|c|}{ I04166 } \\
\hline & $\mathrm{X}_{4}^{\mathrm{CO}}$ & $f_{\text {enh }}$ & $\mathrm{X}_{4}^{\mathrm{CO}}$ & $f_{\text {enh }}$ & $\mathrm{X}_{4}^{\mathrm{CO}}$ & $f_{\text {enh }}$ & $\mathrm{X}_{4}^{\mathrm{CO}}$ & $f_{\mathrm{enh}}$ & $\mathrm{X}_{4}^{\mathrm{CO}}$ & $f_{\text {enh }}$ & $\mathrm{X}_{4}^{\mathrm{CO}}$ & $f_{\text {enh }}$ \\
\hline $\mathrm{SiO}^{2}$ & 2.4 & $4.0 \times 10^{3}$ & 6.4 & $1.1 \times 10^{4}$ & 11 & $1.9 \times 10^{4}$ & 1.8 & $3.1 \times 10^{3}$ & 7.3 & $1.2 \times 10^{4}$ & 12.5 & $2.1 \times 10^{4}$ \\
\hline SO & 18 & $2.7 \times 10^{2}$ & 19 & $2.9 \times 10^{2}$ & 19 & $2.9 \times 10^{2}$ & 18 & $2.8 \times 10^{2}$ & 24 & $3.7 \times 10^{2}$ & 23 & $3.5 \times 10^{2}$ \\
\hline $\mathrm{CS}$ & 5.5 & 7.9 & 11 & 17 & 1.2 & 1.7 & 3.2 & 4.7 & 9.3 & 13 & 1.7 & 2.4 \\
\hline $\mathrm{CH}_{3} \mathrm{OH}$ & 79 & $5.7 \times 10^{2}$ & 69 & $5.0 \times 10^{2}$ & 28 & $2.0 \times 10^{2}$ & 80 & $5.8 \times 10^{2}$ & 54 & $3.9 \times 10^{2}$ & 20 & $1.4 \times 10^{2}$ \\
\hline $\mathrm{HC}_{3} \mathrm{~N}$ & 2.0 & 13 & 3.2 & 21 & $<1.8$ & $<11$ & $<3.1$ & $<20$ & $<3.3$ & $<22$ & $<1.7$ & $<10$ \\
\hline $\mathrm{HCO}^{+}$ & 0.93 & 2.0 & 0.95 & 1.9 & 0.88 & 1.8 & 0.4 & 0.8 & 1.3 & 2.5 & 0.9 & 1.9 \\
\hline $\mathrm{p}-\mathrm{H}_{2} \mathrm{CO}^{3}$ & 1.9 & 27 & 1.0 & 14 & 0.40 & 5.5 & 2.8 & 39 & $<0.4$ & $<5.7$ & $<1.4$ & $<22$ \\
\hline $\mathrm{HCN}$ & 9.7 & 9.1 & 22 & 20 & 2.1 & 2.0 & 4.9 & 4.6 & 23 & 21 & 0.88 & 0.82 \\
\hline
\end{tabular}

Notes. ${ }^{(1)} \mathrm{X}_{4}^{\mathrm{CO}}=10^{4} \times N_{T} / \mathrm{N}(\mathrm{CO})$, and $f_{\text {enh }}$ as defined in Sect. 4.3 ; ${ }^{(2)}$ the assumed $\mathrm{SiO}$ abundance in cores is the upper limit suggested by Ziurys et al. (1989): $5 \times 10^{-12}$; ${ }^{(3)}$ an ortho-to-para ratio of 3 was assumed for $\mathrm{H}_{2} \mathrm{CO}$.

abundances in all the outflow regimes are those of $\mathrm{CH}_{3} \mathrm{OH}$, with values up to almost $10^{-2}$ in the wings. The next most abundant species are $\mathrm{SO}, \mathrm{HCN}, \mathrm{CS}$, and $\mathrm{SiO}$, with values of a few times $10^{-3}$, also in the wing regimes. $\mathrm{HC}_{3} \mathrm{~N}$, para- $\mathrm{H}_{2} \mathrm{CO}$, and $\mathrm{HCO}^{+}$, on the other hand, present the lowest measured abundances in our survey, with relative values respect to $\mathrm{CO}$ on the order of $10^{-4}$.
Given the order-of-magnitude difference in energetics and central source luminosity between the L1448 and I04166 outflows, we start our chemistry analysis by comparing the abundance results for the two objects. This comparison is illustrated in Fig. 7 with the L1448/I04166 ratio of CO-normalized abundances for each outflow regime and for each molecular species detected in both sources. As it can be seen, when the abundances 


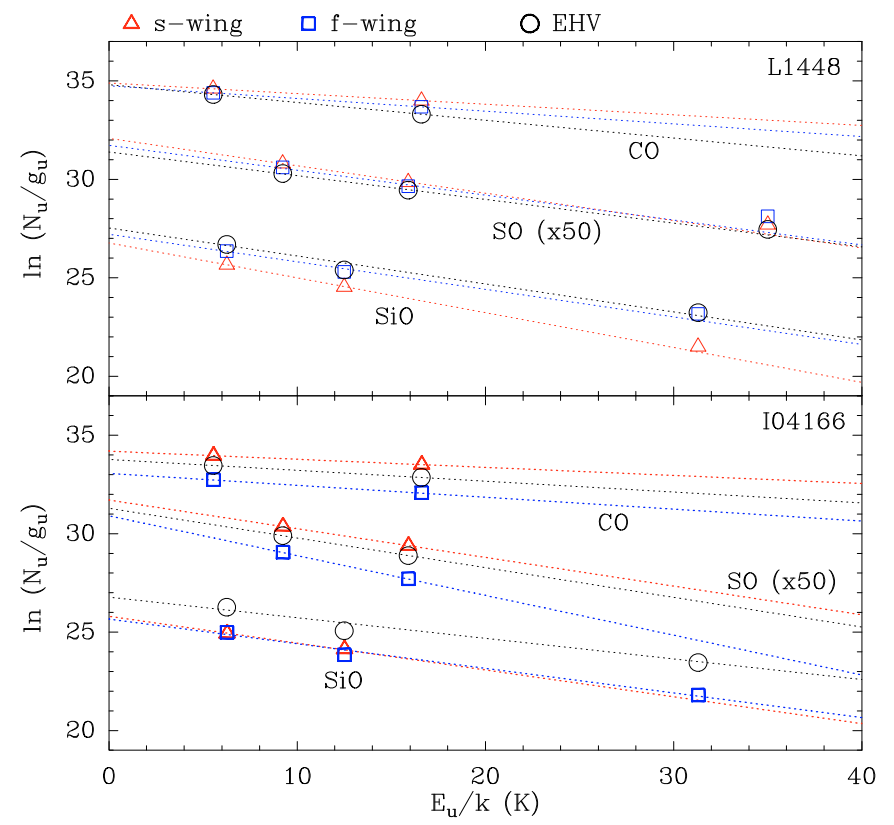

Fig. 6. Population diagrams for CO, SiO, and SO in L1448 and I04166 illustrating the results from the excitation analysis. Red triangles, blue squares, and black circles represent s-wing, f-wing, and EHV values, respectively. The lines show the results from the least-squares fit described in the text and summarized in Table 3. The SO data have been multiplied by 50 for presentation purposes only.

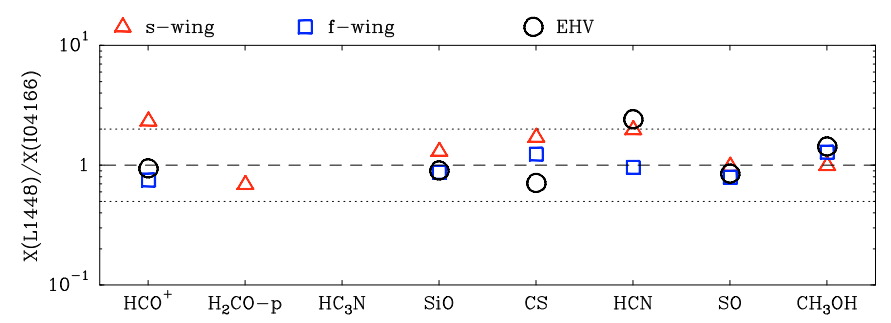

Fig. 7. Ratio between the CO-normalized abundances derived for L1448 and I04166 in each outflow regime (symbols as in Fig. 6). Note how the points cluster around a value of 1 . Only detections have been used. No $\mathrm{HC}_{3} \mathrm{~N}$ data are presented due to its non detection in the I04166 outflow (at levels consistent with an abundance ratio of 1 , see Table 3 ).

are compared in the same outflow regime, all ratios are clustered around 1 and show no clear deviation from that value, independent on the species or the outflow velocity regime. The mean value of the ratios in the sample is 1.2 and the rms is 0.5 , which suggest that the abundances are very close, or equal, in the two sources. This is a first indication that the abundances determined from our survey may represent "typical" values in outflows from Class 0 sources, and not be just peculiar to L1448 and I04166. The comparison with the L1157 outflow abundances in Sect. 4.4 will further strengthen this interpretation.

\subsection{Chemical differences between the outflow regimes}

The analysis of the spectra in Sect. 3.2 already suggested that species like $\mathrm{CH}_{3} \mathrm{OH}$ and $\mathrm{H}_{2} \mathrm{CO}$ favor low velocities, while species like $\mathrm{SiO}$ are enhanced in the EHV gas. Table 3 shows quantitatively that these trends are real, and that the abundance of most molecules varies systematically between the different outflow regimes. To illustrate these abundance variations, we compare in Fig. 8 the CO-based abundances of several molecules normalized to a value of 1 in the s-wing regime. As the left panel shows, the abundance of $\mathrm{CH}_{3} \mathrm{OH}$ and $\mathrm{SiO}$ follow opposite trends across the three outflow regimes. If the abundances of these species are normalized to 1 in the s-wing regime, they differ by a factor of 20 in the EHV gas. The SO abundance, on the other hand, remains approximately constant across the three outflow regimes, indicating that this species has either constant abundance or that any abundance variation follows closely that of $\mathrm{CO}$. As the figure also shows, the abundance variations between the outflow velocity regimes are very similar in L1448 (squares) and I04166 (triangles), illustrating again the consistent behavior of the chemistry in the two outflows.

In contrast with the monotonic changes with velocity seen in $\mathrm{SiO}, \mathrm{SO}$, and $\mathrm{CH}_{3} \mathrm{OH}$, the abundance of other species presents more complex patterns. $\mathrm{CS}$ and $\mathrm{HCN}$, shown in the right panel of Fig. 8, have significant (factor of a few) enhancements between the s-wing and f-wing regimes, but they are strongly underabundant in the EHV gas. As the figure shows, HCN presents the most extreme change in the EHV regime, and its abundance drops by more than one order of magnitude respect to the f-wing value in both L1448 and I04166 (even assuming that the winglike $\mathrm{HCN}$ emission we observe truly represents EHV gas). This abundance behavior of $\mathrm{CS}$ and $\mathrm{HCN}$ indicates that the f-wing and the EHV regimes have rather independent chemical compositions, and that molecules cannot be simply characterized as favoring high or low velocities across the whole outflow range.

Further illustration of the chemical differences between the outflow regimes is shown in Fig. 9, where we compare the L1448 spectra for $\mathrm{HCN}$ and $\mathrm{SiO}$. These species follow a similar abundance increase between the s-wing and f-wing regimes, but they behave very differently in the EHV gas. To properly compare the spectra, we have used the $\mathrm{SiO}(2-1)$ line as a template to model the $\mathrm{HCN}(1-0)$ hyperfine triplet, as discussed in the Appendix $\mathrm{C}$, and we have matched the intensities of the two lines for the f-wing regime. Given the similar frequencies of $\mathrm{SiO}(2-1)$ and HCN(1-0) (Table 1), their beam-dilution factors are approximately equal, so the different velocity regimes can be compared directly from the spectra without additional scaling factors. As can be seen in the figure, the spectra match well inside the f-wing regime, but the two lines strongly diverge from each other in the EHV gas. SiO presents a bright EHV peak, while HCN shows a weak wing. As discussed in Sect. 3.4, the HCN wing could be a continuation of the f-wing regime and not a separate EHV feature, so it is difficult to estimate the exact abundance contrast between the two species in the EHV regime. Comparing the intensities of the two spectra in the fastest second half of the EHV regime (to minimize wing contamination), we estimate that the $\mathrm{SiO} / \mathrm{HCN}$ abundance ratio in the $\mathrm{EHV}$ gas is at least a factor of 20 lower than in the f-wing regime, and the factor could be higher if the high velocity wing in $\mathrm{HCN}$ is still part of the f-wing gas and does not belong to the EHV regime. Such a large difference in abundance suggests that the mechanism causing the enhancement of $\mathrm{HCN}$ in the f-wing regime is not operating in the EHV gas, and that a combination of several chemical processes is required to explain the abundances observed across the outflow regimes. Before investigating possible processes behind the observed behavior, it is convenient to compare the abundances measured in the L1448 and I04166 outflows with those already determined for different environments.

\subsection{Comparison with dense core abundances}

We first compare the outflow abundances with those expected for the surrounding ambient gas. Ideally, we should use the ambient 


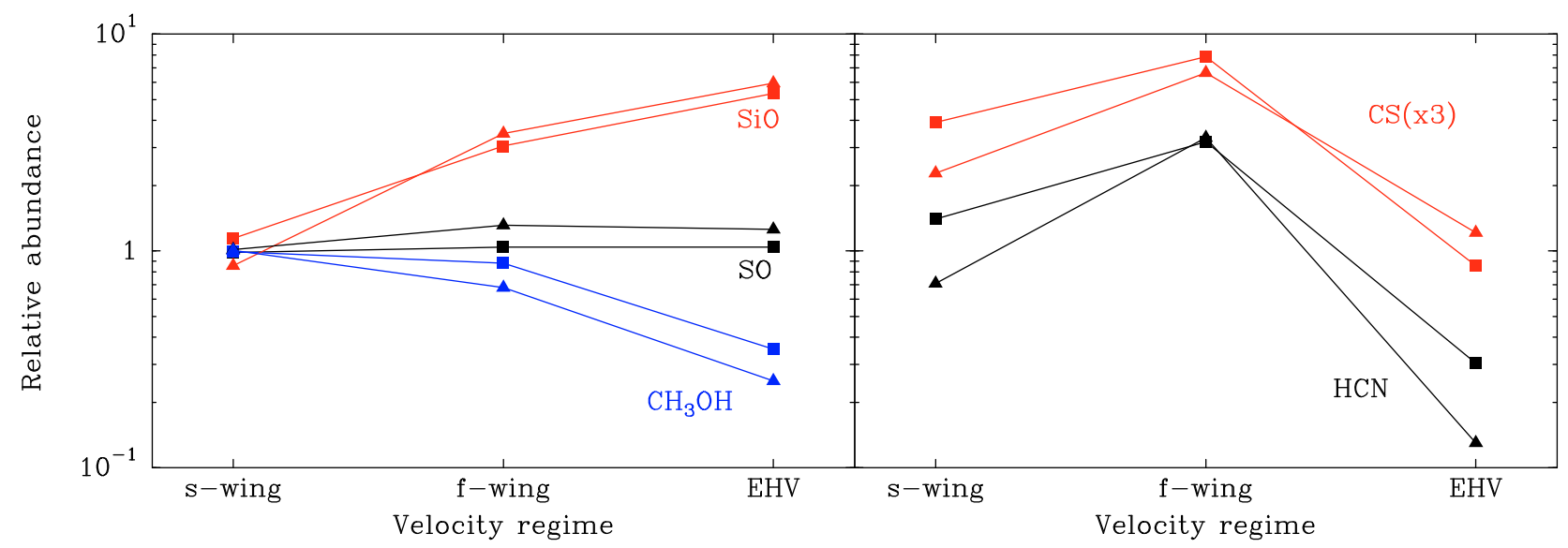

Fig. 8. Variation of CO-normalized abundances over the three outflow regimes. Values are set to unity in the s-wing regime to better illustrate their change across the outflow. Squares represent L1448 data and triangles represent I04166 data. Note the good agreement between the two outflows and the systematic differences between the species. The CS data have been multiplied by 3 to avoid overlap with the HCN curve.

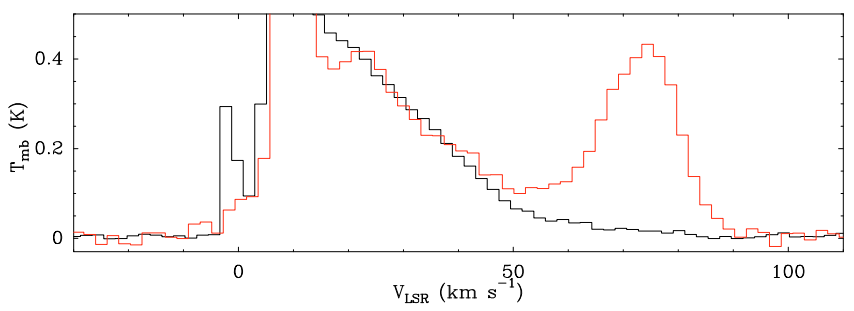

Fig. 9. Comparison between the HCN(1-0) spectrum (black) in L1448 and a synthetic spectrum generated using $\mathrm{SiO}(2-1)$ as a template (red). Note the good match between the two histograms in the f-wing and the large difference in the EHV regime. This change of behavior indicates a higher (factor of 20) change in the $\mathrm{SiO} / \mathrm{HCN}$ abundance ratio between the f-wing and the EHV regime.

line components in the outflow survey spectra to infer the composition of the dense gas surrounding L1448-mm and I04166. Unfortunately, this is not possible because the ambient components suffer from optical depth effects and contamination from the reference position in the "wobbler switch" observing mode. As an alternative, we compare the outflow abundances with those of similar dense cores for which a reliable abundance determination exists. Two good sources for this comparison are the starless cores L1498 and L1517B in the Taurus-Auriga molecular cloud (to which I04166 belongs), whose composition has been determined in detail using multi-line radiative transfer by Tafalla et al. (2004a, 2006). Like most starless cores, L1498 and L1517B suffer from strong molecular depletion at their centers due to freeze out onto dust grains. For the comparison with the outflow abundances, we use the undepleted abundances characteristic of the core outer parts, which most likely correspond to the pre-shock ambient abundances for the positions in our survey. As the abundances in L1498 and L1517B are similar, we take their geometric mean and use the result as our reference value for the comparison. We define an enhancement factor $f_{\text {enh }}$ for each species as the ratio between the outflow abundance and the reference core abundance, and present the result for each outflow range in Table 4.

Figure 10 shows a plot of the $f_{\text {enh }}$ factor for each outflow regime in L1448 and I04166. As already mentioned, the L1448 and $\mathrm{I} 04166$ results agree with each other for all species and outflow regimes for which we have measurements, and from now on we will ignore source to source variations and concentrate on a few general trends (we consider that factor-of- 2 differences are

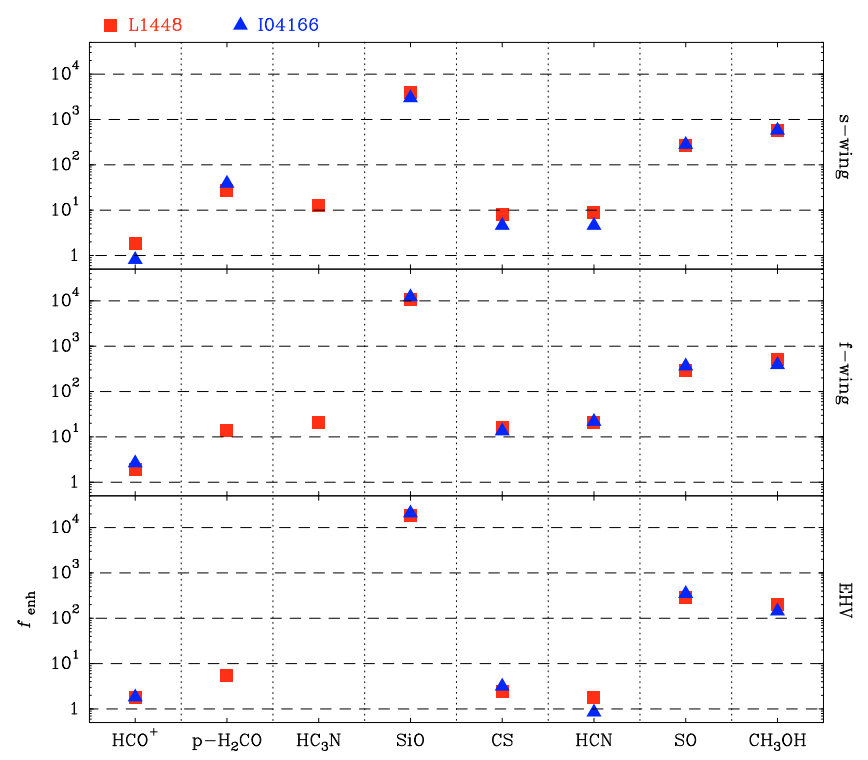

Fig. 10. Abundance enhancement factors with respect to dense core values for the three outflow regimes of L1448 (red squares) and I04166 (blue triangles). Note how all abundances, with the possible exception of $\mathrm{HCO}^{+}$, are significantly (order of magnitude) enhanced in most outflow regimes. Note also the lower enhancement of species like CS and $\mathrm{HCN}$ in the EHV gas.

at the limit of significance). Overall, most $f_{\text {enh }}$ factors shown in Fig. 10 are larger than unity, indicating that the outflow abundances exceed systematically the abundances in starless cores. As can be seen, the two wing regimes present similar patterns of enhancement, having the f-wing gas higher enhancement factors than s-wing in all species but $\mathrm{CH}_{3} \mathrm{OH}$ and $\mathrm{p}-\mathrm{H}_{2} \mathrm{CO}$. The pattern of enhancements in the EHV gas, on the other hand, is clearly different from that in the wings, especially concerning CS and HCN. As discussed in Sect. 4.2, these species are significantly enhanced in both s-wing and f-wing regimes, but their abundance exceeds the core values only marginally in the EHV gas.

As the figure shows, the enhancement factors in the outflow gas span 4 orders of magnitude. $\mathrm{HCO}^{+}$is the least enhanced molecule in our survey, showing an overabundance of at most a factor of 2 with respect to the dense cores, and has only marginal variations between the different outflow regimes. A second group of molecules consists of $\mathrm{p}-\mathrm{H}_{2} \mathrm{CO}, \mathrm{HC}_{3} \mathrm{~N}, \mathrm{CS}$, 
and $\mathrm{HCN}$, which present abundance enhancements of about one order of magnitude in the two wing regimes. Interestingly, three of these species show lower enhancement factors in the EHV gas, while the case for $\mathrm{HC}_{3} \mathrm{~N}$ is unclear since its non detection in the EHV regime imposes only a very weak constraint. An additional order of magnitude increase in the abundance enhancement factor is found for $\mathrm{SO}$ and $\mathrm{CH}_{3} \mathrm{OH}$. As seen in the previous section, the $\mathrm{SO}$ factor remains almost constant over the whole outflow range (at a level of about 300), while the $\mathrm{CH}_{3} \mathrm{OH}$ factor gradually drops by about 3 from the s-wing gas to the EHV regime. Finally, $\mathrm{SiO}$ presents the most extreme enhancement factors of the sample. The $\mathrm{SiO} f_{\text {enh }}$ factor increases by more than 5 between the s-wing and the EHV regimes, and has a typical value in the f-wing gas of about $10^{4}$, with the caveat that the non detection of $\mathrm{SiO}$ in starless cores makes this enhancement factor a somewhat uncertain lower limit (Ziurys et al. 1989).

The above overabundance of most molecules in the outflow gas indicates that this material has been strongly processed chemically with respect to the more quiescent gas around it. This is particularly evident in the wing component, which most likely arises from gas initially in the ambient core and that has been shock-accelerated by the outflow wind. Indeed, the pattern of abundances in the wing components of the outflows bears strong similarities with that of the L1157 outflow, as we will now see.

\subsection{Comparison with the L1157 outflow}

The L1157 outflow is commonly considered the reference source when studying the chemistry of low-mass outflows. It is powered by a $11 L_{\odot}$ Class 0 source (Umemoto et al. 1992), and its composition presents high molecular abundance enhancements with respect to the surrounding medium suggestive of a strong shock chemistry in action (Bachiller \& Pérez Gutiérrez 1997). To compare the outflow abundances of L1448 and I04166 with those of L1157, we have re-analyzed the data of Bachiller \& Pérez Gutiérrez (1997) for the so-called B1 position (the brightest peak in the blue lobe) using the same methodology applied in Sect. 3.5 to the L1448 and I04166 data. The results of this new analysis are not very different from those in the original paper, and are presented with more detail in Appendix D. As the L1157 outflow lacks an EHV component, a proper comparison with the L1448 and I04166 outflows should be done using the wing regime. For L1448 and I04166, we chose the f-wing regime, since in this range the abundance of most species remains constant with velocity, and because for molecules with complex spectra like $\mathrm{HCN}$ and $\mathrm{CH}_{3} \mathrm{OH}$, the abundances in the f-wing regime are better determined than in the s-wing regime, which is often confused by line overlaps. For L1157-B1, we define an f-wing component taking the fastest half of the wing emission, following a similar criterion to that used to define the f-wing regime in L1448 and I04166.

Figure 11 presents a comparison between the CO-normalized abundance of all the species observed both in L1157-B1 and our two outflow targets. As it can be seen, the abundance of most species agrees better than a factor of 2 in the three outflows, being the only exceptions $\mathrm{p}-\mathrm{H}_{2} \mathrm{CO}$ and $\mathrm{CH}_{3} \mathrm{OH}$, which are more abundant in L1157. This general agreement between the L1448 and I04166 abundances and those of L1157-B1 is remarkable given the almost 2 orders of magnitude spanned by the values in the figure and by the several orders of magnitude of enhancement with respect to the ambient values that these abundances represent. It is also remarkable because the L1157 outflow is very different in

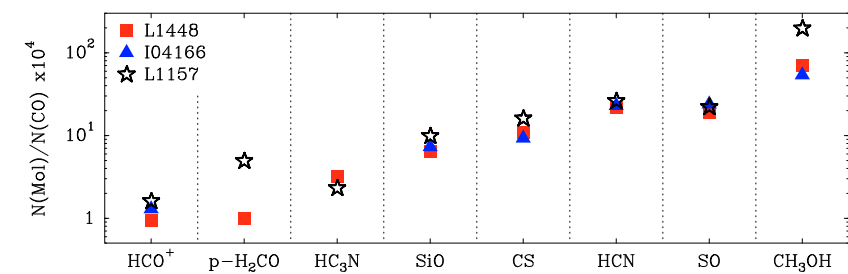

Fig. 11. Comparison between CO-normalized outflow abundances in L1448 (red squares), I04166 (blue triangles) and L1157 (open stars) for all species detected in the molecular survey. Note the good agreement (better than a factor of two) for most species. See text for a discussion on the discrepancies in $\mathrm{H}_{2} \mathrm{CO}$ and $\mathrm{CH}_{3} \mathrm{OH}$. For all sources, $\mathrm{f}$-wing regime values have been used.

physical properties from the L1448 and I04611 outflows. In addition to lacking EHV gas, L1157 presents a combination of a low velocity range and a high $\mathrm{CO}$ column density that makes its CO column density per $\mathrm{km} \mathrm{s}^{-1}$ higher than that of L1448 and I04166 by factors 13 and 36, respectively. The good matching between abundances shown in Fig. 11 therefore indicates that outflows with different physical properties, but still powered by Class 0 sources, can have very similar chemical compositions. It also indicates that the abundances do not depend strongly on the environment where the source has formed, as L1448 and I04166 lie in large clouds (Perseus and Taurus), while L1157-mm was formed in a more isolated environment. We take this good match as a suggestion that the abundances determined in this analysis are not peculiar to the sources in the sample, and that they likely represent a pattern common to other Class 0 outflows.

The similar abundances found in L1448, I04166, and L1157 do not imply that there is perfect chemical uniformity. L1157 itself presents factor-of- 2 abundance differences between its several well studied positions (B1 versus B2, see Bachiller \& Pérez Gutiérrez 1997; Rodríguez-Fernández et al. 2010), and high angular resolution observations of the $\mathrm{B} 1$ region reveal internal composition gradients (Benedettini et al. 2007; Codella et al. 2009). In addition, Fig. 11 shows that the agreement between the abundances in L1448, I04166, and L1157 is not perfect. Even after ignoring abundance differences smaller than a factor of 2 (that could arise from uncertainties in the analysis), discrepancies remain in the abundances of $\mathrm{p}-\mathrm{H}_{2} \mathrm{CO}$ and $\mathrm{CH}_{3} \mathrm{OH}$. The factor of 5 discrepancy in the abundance of $\mathrm{p}-\mathrm{H}_{2} \mathrm{CO}$, however, should be treated with some care. For L1157, only ortho $\mathrm{H}_{2} \mathrm{CO}$ has been measured, while for L1448 and I04166, only the para form was observed, and it was necessary to assume an ortho-to-para ratio for the comparison (the high-temperature limit of 3 was used). Additionally, our $\mathrm{H}_{2} \mathrm{CO}$ abundance determination is based on a single lines, and it can be uncertain due to NLTE excitation in the irregularly-spaced $\mathrm{H}_{2} \mathrm{CO}$ energy ladder. The factor of 3 higher $\mathrm{CH}_{3} \mathrm{OH}$ abundance in $\mathrm{L} 1157$, on the other hand, seems better established, as the same transitions were used in all sources. Interestingly, both $\mathrm{H}_{2} \mathrm{CO}$ and $\mathrm{CH}_{3} \mathrm{OH}$ are the two molecules that favor low velocities in L1448 and $\mathrm{I} 04166$ $\left(\mathrm{H}_{2} \mathrm{CO}\right.$ is significantly more abundant in the s-wing regime), and they also present a similar behavior in L1157-B1 (where $\mathrm{CH}_{3} \mathrm{OH}$ is slightly more enhanced than $\mathrm{H}_{2} \mathrm{CO}$ in the slower gas). Such velocity dependence of the abundance could in principle explain the higher values measured towards L1157, as the gas in this outflow moves slower than the gas in L1448 and I04166. Further observations of $\mathrm{H}_{2} \mathrm{CO}$ and $\mathrm{CH}_{3} \mathrm{OH}$ are needed to test this hypothesis, and a more detailed study of the chemistry of these two species, using a large sample of outflows, will be presented in Santiago-García et al. (2010, in preparation). 


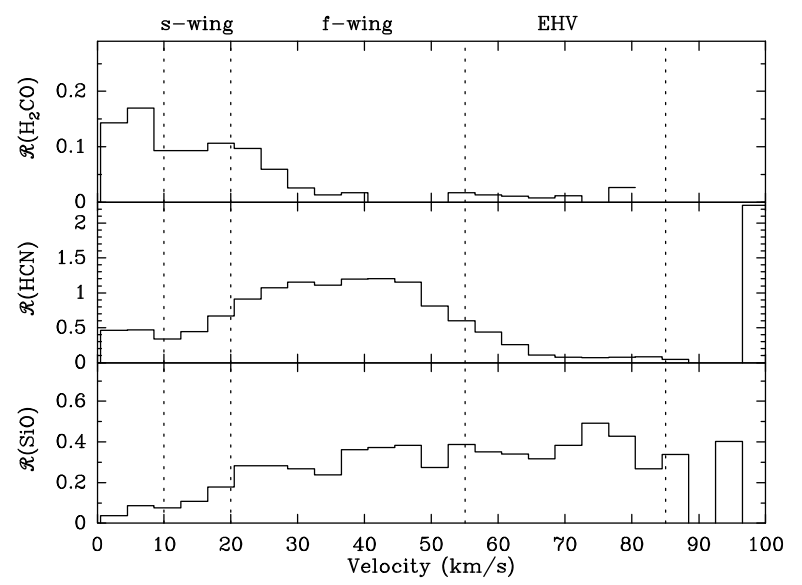

Fig. 12. Intensity ratio with respect to $\mathrm{CO}$ in $\mathrm{L} 1448$ for three species with different behavior across the outflow regimes. Note how $\mathrm{H}_{2} \mathrm{CO}$ favors the s-wing, $\mathrm{HCN}$ favors the f-wing, and $\mathrm{SiO}$ is mostly present in the f-wing and EHV gas (with a slight increase in the EHV regime).

Even after considering the above differences among outflows (and between parts of the same outflow in the case of L1157), the main pattern that emerges from Fig. 11 is that of relative (factor of 2) homogeneity between the composition of the different outflow wings. As further discussed in the next section, that composition most likely arises from shock chemistry, and many of the observed variations are likely to result from the natural dependence of this chemistry on the shock velocity. In that sense, L1448, I04166, and L1157 seem representative of a chemically rich early phase in the life of a bipolar flow.

\section{Nature of the outflow regimes and their chemical composition}

\subsection{Origin of the observed outflow velocities}

Before discussing the origin of the different outflow regimes, it is worth noting that the presence of these regimes provides by itself useful information on the outflow velocity field. Line spectra only provide information on the radial component of the gas velocity field, so they do not reveal the full 3D picture of the gas motions. Outflow wings, in particular, can arise from a variety of velocity fields, of which there are two extreme cases (Margulis \& Lada 1985): (i) the gas has a single speed and the different radial velocities in the spectra arise solely from projection effects; and (ii) the gas has a true distribution of speeds, and the observed radial velocities faithfully reflect that distribution. The presence of velocity-dependent molecular abundances can help distinguish between these two extreme cases, as molecular composition, in contrast to velocity, is projection independent. To illustrate this idea, we present in Fig. 12 the velocity-dependent emission ratio with respect to $\mathrm{CO}$ of three species, $\mathrm{H}_{2} \mathrm{CO}, \mathrm{HCN}$, and $\mathrm{SiO}$. As can be seen, each ratio depends on velocity in a different way: $\mathrm{H}_{2} \mathrm{CO}$ is more abundant in the s-wing regime, $\mathrm{HCN}$ dominates the f-wing regime, and $\mathrm{SiO}$ does so over both the f-wing and EHV gas, in agreement with the results of previous sections. This variety of behaviors, together with the close-toconstant excitation conditions derived in Sect. 3.5, indicates that the emitting gas cannot consist of a single, homogeneous gas component whose distribution in radial velocities is only due to projection effects. The observed changes in the gas composition as a function of velocity imply necessarily that the velocity regimes we observe are intrinsically distinct, and that the radial velocities must represent in some way the true (3D) velocities of the gas.
The idea that projection effects in a constant-velocity gas cannot explain the observed outflow wings also agrees with the high collimation of the L1448 and I04166 outflows. Interferometer observations of the flows show that their opening angle near the YSO ranges from $30^{\circ}$ to $45^{\circ}$, and that the collimation at large distances is even higher than that (Bachiller et al. 1995; Santiago-García et al. 2009). Projection effects from such a narrow range of angles seem insufficient to explain the high velocity span of the outflow wings $\left(\sim 50 \mathrm{~km} \mathrm{~s}^{-1}\right.$ in L1448 and $\sim 30 \mathrm{~km} \mathrm{~s}^{-1}$ in I04166). Thus, in the following discussion we will assume that the observed radial velocities are proportional to the 3D gas speed of the flow. The proportionality factor, unfortunately, is rather uncertain, as the inclination angle of the outflows with respect to the line of sight is not well constrained. For L1448, Girart \& Acord (2001) used a 2-epoch measurement of the proper motions of the EHV SiO emission peaks to estimate an inclination angle with respect to the plane of the sky of $21^{\circ}$, which implies a true EHV velocity of about $180 \mathrm{~km} \mathrm{~s}^{-1}$. Although possible, the projection correction implied by this measurement seems very high, as the EHV component in L1448 already has one of the highest radial velocities observed, and with the correction, L1448 becomes an extreme object among its class. Lacking an alternative measurement for I04166, and to avoid a possible overcorrection factor, we will assume in the following discussion a representative projection angle of $45^{\circ}$ for both L1448 and I04166. We remark, however, that a larger correction will not invalidate the discussion below. In fact, it will make the problems of the chemical models discussed to explain the EHV emission even more acute than we estimate.

\subsection{Two outflow wing regimes: velocity-dependent shock chemistry}

As discussed before, the chemical composition of the outflow wings in L1448 and I04166 is very similar to that of the L1157 outflow, which is often considered a textbook example of shock chemistry (Bachiller \& Pérez Gutiérrez 1997; Benedettini et al. 2007; Codella et al. 2009; Rodríguez-Fernández et al. 2010). In addition, the gas in the outflow wings is commonly interpreted as resulting from accelerated ambient gas (e.g., Santiago-García et al. 2009), and an acceleration to the observed supersonic velocities necessarily implies the presence of a shock. Thus, it seems natural to interpret the wing abundances in L1448 and I04166 in terms of shock chemistry, and to try to explain the composition differences between the s-wing and f-wing gas as a result of the velocity dependence expected for this type of chemistry.

In Sect. 4.2 (see also Fig. 12) we saw that the s-wing regime has lower $\mathrm{SiO}$ and $\mathrm{HCN}$ abundances than the f-wing gas, while it is relatively enhanced in $\mathrm{H}_{2} \mathrm{CO}$ and $\mathrm{CH}_{3} \mathrm{OH}$. These latter molecules are often referred to as "primary" species, because their abundance enhancement in outflows and warm gas is thought to occur via their direct release from the mantles of dust grains (e.g., Charnley et al. 1992). In outflows, such a release is often explained as the result from the sputtering of the (electrically charged) dust grains as they stream through the neutral gas inside a C-type shock (Draine et al. 1983). As the high $\mathrm{H}_{2} \mathrm{CO}$ and $\mathrm{CH}_{3} \mathrm{OH}$ abundances in the s-wing gas indicate, grain mantle destruction must be occurring even at the relatively low velocities characteristic of this outflow regime, whose lower limit we estimate in $5 \mathrm{~km} \mathrm{~s}^{-1}$ assuming a inclination angle of $45^{\circ}$ for both the L1448 and I04166 outflows. Such low velocity starts to be uncomfortable for grain sputtering models. The 
classical analysis by Draine et al. (1983) predicts that most water ice is returned to the gas phase due to sputtering for shocks faster than $25 \mathrm{~km} \mathrm{~s}^{-1}$, and although the number has been recently lowered slightly by Jiménez-Serra et al. (2008) for the release of $\mathrm{CH}_{3} \mathrm{OH}$ and $\mathrm{H}_{2} \mathrm{O}$, it is still on the order of $20 \mathrm{~km} \mathrm{~s}^{-1}$. (A much lower value, around $10 \mathrm{~km} \mathrm{~s}^{-1}$ was proposed for dirty ices by Flower \& Pineau des Forets (1994), but the value of the sputtering threshold used by these authors is uncertain.) Requiring that our observed minimum s-wing radial velocity corresponds to the high speed of the above models would imply that both outflows are less than $15^{\circ}$ from the plane of the sky, which seems unlikely given the already very high (radial) velocity of their EHV gas. On the other hand, although it is possible that the outflow gas has slowed down significantly from its initial shock velocity, Fig. 12 shows that the $\mathrm{SiO}$ and $\mathrm{HCN}$ abundances drop in the s-wing regime compared to the f-wing regime. This suggests that only little gas from the f-wing regime has been transferred to the s-wing part of the flow.

A possible solution to the observed low-velocity enhancement in $\mathrm{CH}_{3} \mathrm{OH}$ and $\mathrm{H}_{2} \mathrm{O}$ is to invoke an additional process of mantle disruption, like direct collisions between dust grain particles (Tielens et al. 1994). Caselli et al. (1997) have shown that grain-grain collisions in oblique C-type shocks can lead to complete water ice evaporation at shock velocities as low as $15 \mathrm{~km} \mathrm{~s}^{-1}$. These authors, in addition, suggest that the release of less bound species like $\mathrm{CH}_{3} \mathrm{OH}$ could occur at velocities as low as $10 \mathrm{~km} \mathrm{~s}^{-1}$, in better agreement with our observations. Clearly more work is needed to understand low-velocity mantle disruption, which is not peculiar from the sources of our survey, but seems occur with relatively high frequency (e.g., Garay et al. 2002).

In contrast with the s-wing regime, the f-wing gas is characterized by an abundance drop of $\mathrm{H}_{2} \mathrm{CO}$ (and to a less extent $\left.\mathrm{CH}_{3} \mathrm{OH}\right)$ together with an enhancement of $\mathrm{SiO}, \mathrm{HCN}$, and $\mathrm{CS}$ (Fig. 12). This composition change occurs at a velocity of approximately $20 \mathrm{~km} \mathrm{~s}^{-1}$ for both L1448 and I04166, again assuming an outflow inclination angle of $45^{\circ}$. As illustrated by the Fig. 12, the composition change between s-wing and f-wing regimes is relatively sudden, especially taking into account that after the transition, the abundance of most species remains almost constant for about 20 to $30 \mathrm{~km} \mathrm{~s}^{-1}$ (up to the EHV regime). Such a relatively sharp transition between the s-wing and $\mathrm{f}$-wing regimes suggests that one or more additional chemical processes start to operate in the gas at that velocity, and that they continue to operate for the rest of the f-wing regime. To our knowledge, no current chemical model can explain the changes we observe. The abundance decrease of $\mathrm{H}_{2} \mathrm{CO}$ and $\mathrm{CH}_{3} \mathrm{OH}$, for example, suggest that these species are being destroyed at high velocities either during grain sputtering or via gas-phase reactions, while mantle sputtering and grain-grain collision models commonly predict a steady increase in abundance with velocity (e.g., Caselli et al. 1997; Jiménez-Serra et al. 2008). The enhancement of $\mathrm{SiO}$, on the other hand, has been modeled in detail by a number of authors, and two alternative scenarios have been usually explored: release of silicon from grain cores and from grain mantles (Schilke et al. 1997; Caselli et al. 1997; Gusdorf et al. 2008a,b). The sudden abundance increase of SiO near $20 \mathrm{~km} \mathrm{~s}^{-1}$ could in principle be interpreted as indicating the crossing of the threshold for core grain disruption (although a value close to $30 \mathrm{~km} \mathrm{~s}^{-1}$ would have been expected, Gusdorf et al. 2008a). Still, the relatively constant $\mathrm{SiO}$ abundance in the f-wing gas after crossing that threshold is puzzling, since models predict a steady increase of the $\mathrm{SiO}$ yield with velocity once grain core erosion starts. Thus, although our observed f-wing abundances are likely to result from shock chemistry, and the values measured towards L1448 and I04166 agree well with each other (and with those in L1157), no current model seems to explain all the observed trends.

\subsection{The peculiar composition of the EHV component}

The composition of the EHV gas has remained poorly known due to the lack of data apart from $\mathrm{CO}$ and $\mathrm{SiO}$. The detection of $\mathrm{SO}, \mathrm{CH}_{3} \mathrm{OH}$, and $\mathrm{H}_{2} \mathrm{CO}$, together with the less clear detections or upper limits for $\mathrm{HCO}^{+}, \mathrm{CS}$, and $\mathrm{HCN}$, make it possible now to explore the chemistry of this gas component in more detail. As discussed in Sect. 4.2, the EHV gas partly continues the trend of velocity-dependent abundances found in the wing components: $\mathrm{SiO}$ becomes more enhanced, $\mathrm{SO}$ (and probably $\mathrm{HCO}^{+}$) remain almost constant, and $\mathrm{H}_{2} \mathrm{CO}$ and $\mathrm{CH}_{3} \mathrm{OH}$ drop slightly with respect to the wing gas. CS and $\mathrm{HCN}$, on the other hand, deviate strongly from this behavior. The two species are significantly ( $\approx$ factor of 2 ) enhanced in the f-wing regime compared to the s-wing gas, but the transition to the EHV gas represents an abundance drop of about one order of magnitude. The most extreme example of this behavior is HCN in L1448, due to its high signal-to-noise ratio (Figs. 9 and 12). Even assuming that the weak, wing-like HCN feature that we observe at the highest velocities corresponds to the EHV gas and is not the (more likely) continuation of the f-wing regime, the HCN abundance in the EHV component is a factor of 10-20 lower than in the f-wing gas. This drop is so high that any $\mathrm{HCN}$ enhancement over the ambient abundance observed in the wing regime seems to have disappeared in the EHV gas (Fig. 10).

The CS and HCN abundance drop between the f-wing and EHV regimes represents the highest contrast seen in our survey, and suggests a significant chemical difference between these two outflow regimes. HCN and CS have in common that their enhancement in shocked gas depends on the presence of carbon in the gas phase. $\mathrm{HCN}$ is mainly produced in shocks by the reaction of $\mathrm{N}$ with $\mathrm{CH}_{2}$, while $\mathrm{CH}_{2}$ itself originates from the reaction of $\mathrm{C}$ with $\mathrm{H}_{3}^{+}$or $\mathrm{C}^{+}$with $\mathrm{H}_{2}$ (Pineau des Forêts et al. 1990; Boger $\&$ Sternberg 2005). CS, on the other hand, is enhanced in shocks by the reaction of $\mathrm{SO}$ and $\mathrm{C}$, which like the previous reactions, has no activation barrier (Pineau des Forêts et al. 1993). The fact that $\mathrm{HCN}$ and CS can be enhanced in the wing gas but not in the EHV component suggests that abundance of free $\mathrm{C}$ in the EHV gas is lower than in the wing. Such a situation could occur if most of the $\mathrm{C}$ in the EHV gas has been quickly locked up into $\mathrm{CO}$, leaving behind little free $\mathrm{C}$ for additional reactions. If this were so, we would expect the EHV gas to contain a relative excess of oxygen. Indeed, Figs. 3 and 5 show that all molecules with prominent $\mathrm{EHV}$ feature are O-bearing species: $\mathrm{CO}, \mathrm{SiO}$, $\mathrm{SO}, \mathrm{CH}_{3} \mathrm{OH}$, and $\mathrm{H}_{2} \mathrm{CO}$. (Note also that $\mathrm{O}$ also destroys $\mathrm{CH}_{2}$, the precursor of $\mathrm{HCN}$, Boger \& Sternberg 2005). We take this peculiar composition of the EHV gas as evidence that this component is richer in oxygen (or poorer in carbon) than the other outflow regimes.

The peculiar C/O abundance ratio in the EHV gas is a potential clue to the nature of this outflow component. As discussed in the introduction, we can classify models of the EHV component as "primary wind" or "shocked ambient gas", depending on whether the material originates from the protostar (or innermost vicinity) or from the surrounding ambient cloud. Unfortunately, no detailed chemical models exist for the composition expected in either interpretation of the EHV gas. Different aspects of the chemistry of shocks and winds, however, have been previously studied, and we can use these results to explore possible 
differences between the two alternative origins of the EHV component. If the EHV gas represents ambient material originally at rest that has been shock-accelerated by a protostellar wind, the speed of the wind has to equal or exceed the velocity of the EHV component. For I04166 and L1448, the radial velocity of the fastest EHV gas with respect to the ambient cloud is 70 and $110 \mathrm{~km} \mathrm{~s}^{-1}$, respectively, again assuming a standard correction for an inclination angle of 45 degrees. Realistic models of molecular shocks in gas at densities of $10^{4} \mathrm{~cm}^{-1}$ or higher predict that for velocities higher than $50-70 \mathrm{~km} \mathrm{~s}^{-1}$, the shock will dissociate $\mathrm{H}_{2}$ and will become of $J$ (jump) type (Draine et al. 1983; Le Bourlot et al. 2002). Any shocks giving rise to the EHV components of L1448 and I04166 must therefore be of $\mathrm{J}$ type, so the species observed in this outflow regime must have reformed from the dissociated material in the postshock gas. Although models of J-type shocks predict efficient reformation of molecules in the postshock gas, they also predict enough UV radiation to photodissociate $\mathrm{CO}$ and keep a relatively high abundance of neutral carbon. The detailed models of J-type shocks by Neufeld \& Dalgarno (1989) show significant $\mathrm{HCN}$ formation in the postshock gas, and predict HCN column densities that are comparable to, and often one order of magnitude higher than those of SO (unfortunately, these authors do not predict CS abundances). Our observations of the EHV gas in L1448 and I04166, however, show a reversed situation, where the SO column density exceeds that of HCN by one order of magnitude or more in the EHV component (abundances in the f-wing gas are comparable in both outflows). This overproduction of HCN (by almost two orders of magnitude with respect to $\mathrm{SO})$ seems to pose a significant challenge to the interpretation of the EHV component as shock-accelerated ambient gas.

To explore the alternative interpretation of the EHV component as a protostellar wind, we use the pioneering work of Glassgold et al. (1991), who studied the composition of a $150 \mathrm{~km} \mathrm{~s}^{-1}$ wind ejected quasi spherically from the surface of a low-mass protostar. (Panoglou et al. 2009 have recently presented an alternative model of a disk wind, but they find no $\mathrm{SiO}$ production, in contrast with observations). Glassgold et al. (1991) found that although the protostellar wind starts atomic in composition, it can become molecular for high enough densities (corresponding to mass-loss rates of a few $10^{-6} M_{\odot} \mathrm{yr}^{-1}$ ). Due to the complexity of the calculation, however, Glassgold et al. (1991) only considered a limited chemical network which did not include the chemistry of SO, HCN, or CS, so we can only make educated guesses on the production rates for these species. Given the relatively high abundance predicted for $\mathrm{OH}$ in the wind, and the lack of activation energy for its reaction with S (Pineau des Forêts et al. 1993), SO formation in the wind seems favorable for the same conditions for which $\mathrm{CO}$ and $\mathrm{SiO}$ are abundant. The possibility of $\mathrm{HCN}$ and CS production, on the other hand, is harder to estimate. It is however noticeable that in the denser models explored by Glassgold et al. (1991) (mass loss rates higher than $3 \times 10^{-6} M_{\odot} \mathrm{yr}^{-1}$ and cases 2 and 3 in the authors notation), all the carbon is locked up into $\mathrm{CO}$ at large radii, and no $\mathrm{C}$ or $\mathrm{C}^{+}$is available for further reactions (their Table 4). This lack of $\mathrm{C}$ suggests that $\mathrm{HCN}$ and CS production may not be favorable for the same wind models that predict abundant $\mathrm{CO}$ and $\mathrm{SiO}$, in agreement with our observations of the EHV gas. (Note however that the model overproduces $\mathrm{SiO}$, but the chemistry of this species is very sensitive to the gas density and the assumed stellar temperature.) In addition to the unexplored chemical paths, the Glassgold et al. (1991) model contains a number of uncertainties that may affect the gas chemistry, like the effect of the higher collimation expected for a jet geometry and the role of the stellar UV field. Still, its chemical paths offer a promising mechanism to explain the observed behavior of $\mathrm{CS}$ and $\mathrm{HCN}$ in the EHV gas.

More challenging for the two scenarios of the origin of the EHV gas is the presence of $\mathrm{CH}_{3} \mathrm{OH}$ and $\mathrm{H}_{2} \mathrm{CO}$ with abundances significantly enhanced with respect to the ambient gas (Sect. 4.3). Gas-phase production of $\mathrm{CH}_{3} \mathrm{OH}$, in particular, is highly inefficient (Geppert et al. 2006), and any abundance enhancement of this species is usually interpreted as resulting from the disruption of dust grain mantles, where $\mathrm{CH}_{3} \mathrm{OH}$ had previously formed via gas-grain reactions (Watanabe et al. 2004). Both shocked-ambient-material and protostellar-wind models, however, need to rely mostly on gas-phase reactions to produce their molecules. In the shocked ambient-gas model, molecules are formed in the post-shock gas due to the dissociative nature of the J-type shock, while in the wind model of Glassgold et al. (1991), the gas starts atomic and hot because it originates near the protostellar surface. Despite this reliance on gas phase reactions, it may be possible for either model to produce $\mathrm{CH}_{3} \mathrm{OH}$ and $\mathrm{H}_{2} \mathrm{CO}$ in the EHV gas by re-forming it on the dust grains via successive hydrogenation of CO (Watanabe et al. 2004). Indeed, both the J-type shock and wind models predict dust grains and abundant atomic hydrogen in the gas phase (Neufeld \& Dalgarno 1989; Glassgold et al. 1991). Unfortunately, no detailed analysis of this chemical path has been studied so far.

As the above discussion illustrates, much information lies still untapped in the chemical composition of the outflow material and in the variations with velocity that this composition suffers. To extract this information, detailed modeling of the chemistry in both shock-accelerated ambient gas and primary winds from protostars and disks is needed. Also needed is a further observational effort to characterize a large sample of outflows in the species found to be sensitive to gas kinematics. Chemical composition, due to its sensitivity to the thermal history of the gas and its initial conditions, offers a unique window to investigate the so-far invisible processes that take place during the production and acceleration of a bipolar outflow.

\section{Conclusions}

We have carried out a molecular survey of one position in each of the outflows powered by L1448-mm and I04166, two Class 0 YSOs in the Perseus and Taurus molecular clouds. These two outflows belong to a special class whose $\mathrm{CO}$ spectra presents a standard outflow wing and a discrete, extremely high velocity (EHV) component, and they are believed to represent one of the earliest phases of the outflow phenomenon. The main results of our study are the following.

1. We have detected a number of molecules in the wings of both outflows, including $\mathrm{CO}, \mathrm{SiO}, \mathrm{SO}, \mathrm{CS}, \mathrm{HC}_{3} \mathrm{~N}, \mathrm{HCO}^{+}, \mathrm{H}_{2} \mathrm{CO}$, $\mathrm{HCN}$, and $\mathrm{CH}_{3} \mathrm{OH}$.

2. In the EHV component, we have detected definitively SO, $\mathrm{CH}_{3} \mathrm{OH}$, and $\mathrm{H}_{2} \mathrm{CO}$, as the spectra from these species present distinct EHV secondary peaks similar to those previously seen in $\mathrm{CO}$ and $\mathrm{SiO}$. In contrast, the spectra from $\mathrm{HCO}^{+}$, $\mathrm{CS}$, and $\mathrm{HCN}$ present low-level wings that overlap with the EHV regime, but the origin of this emission as part of a distinct EHV components is less clear.

3. For most molecules, we have used the intensities of multiple transitions together with a population diagram analysis to derive excitation temperatures and column densities. To compensate for differences in the telescope beam between the different transitions, we have estimated beam-dilution 
factors using high angular resolution $\mathrm{CO}$ maps of the outflows.

4. When normalized to CO, the abundances in the L1448 and I04166 outflows agree with each other better than a factor of 2. This suggests that the abundance patterns we have found may be common to other very young outflows.

5. Based on chemical composition, we identify three different outflow regimes. Two regimes are part of the wing component and the third one consists of the EHV gas. The low-velocity wing regime presents velocity-dependent abundances for most species and is richer than the others in $\mathrm{CH}_{3} \mathrm{OH}$ and $\mathrm{H}_{2} \mathrm{CO}$. The high-velocity wing regime has almost-constant abundance with velocity, and the gas is richer in species like $\mathrm{SiO}, \mathrm{HCN}$, and CS compared to the slower wing. Finally, the EHV gas presents a mixed composition: it has a very high $\mathrm{SiO}$ abundance but is relatively poor in $\mathrm{HCN}$ and CS.

6. The outflow abundances typically exceed those of the Taurus dense cores by one or two orders of magnitude. On the other hand, the fast wing abundances are very similar to those of L1157, the prototypical "chemically active" flow. In agreement with previous work, we interpret the high abundances in the wing components as resulting from shock chemistry. Comparing the slow and fast wing regimes, we find that their composition differences likely originate from the sensitivity of shock chemistry to the velocity of the shock, although no existing model seems capable of explaining all features observed in the data. Our observations illustrate the importance of velocity in the final composition of shock-accelerated gas.

7. Concerning the EHV gas, our molecular survey shows its peculiar chemistry. This gas combines high abundances of O-rich species together with an order of magnitude drop in the abundance of $\mathrm{CS}$ and $\mathrm{HCN}$ with respect to the wing gas. This indicates that the EHV gas has a much lower $\mathrm{C} / \mathrm{O}$ ratio than the shock-accelerated wing gas, and we suggest that it may be a consequence of the two outflow components having different physical origins. One possible explanation of this difference is that the EHV gas represents part of the primary wind from the protostar or its vicinity. Further modeling of the composition of this type of wind is needed to reach a definite conclusion.

Acknowledgements. We thank the staff of the IRAM $30 \mathrm{~m}$ telescope for help during the observations, and Paola Caselli and Eric Herbst for enlightening conversations on shocks and chemistry. This research has made use of NASA's Astrophysics Data System Bibliographic Services and the SIMBAD database, operated at CDS, Strasbourg, France. This work was partially supported by MICINN, within the program CONSOLIDER INGENIO 2010, under grant "Molecular Astrophysics: The Herschel and ALMA era - ASTROMOL" (ref.: CSD2009-00038).

\section{References}

Bachiller, R., \& Pérez Gutiérrez, M. 1997, ApJ, 487, L93

Bachiller, R., \& Tafalla, M. 1999, in The Origin of Stars and Planetary Systems, NATO ASIC Proc, 540, 227

Bachiller, R., Cernicharo, J., Martín-Pintado, J., Tafalla, M., \& Lazareff, B. 1990, A\&A, 231, 174

Bachiller, R., Martín-Pintado, J., \& Fuente, A. 1991, A\&A, 243, L21

Bachiller, R., Guilloteau, S., Dutrey, A., Planesas, P., \& Martín-Pintado, J. 1995, A\&A, 299, 857

Banerjee, R., \& Pudritz, R. E. 2006, ApJ, 641, 949

Bally, J., \& Stark, A. A. 1983, ApJ, 266, L61

Benedettini, M., Viti, S., Codella, C., et al. 2007, MNRAS, 381, 1127

Bergin, E. A., Neufeld, D. A., \& Melnick, G. J. 1998, ApJ, 499, 777
Blake, G. A., Sutton, E. C., Masson, C. R., \& Phillips, T. G. 1987, ApJ, 315, 621 Blake, G. A., Sandell, G., van Dishoeck, E. F., et al. 1995, ApJ, 441, 689 Boger, G. I., \& Sternberg, A. 2005, ApJ, 632, 302

Caselli, P., Hartquist, T. W., \& Havnes, O. 1997, A\&A, 322, 296

Charnley, S. B., Tielens, A. G. G. M., \& Millar, T. J. 1992, ApJ, 399, L71

Chernin, L., Masson, C., Gouveia dal Pino, E. M., \& Benz, W. 1994, ApJ, 426, 204

Codella, C., Benedettini, M., Beltrï¡in, M. T., et al. 2009, A\&A, 507, L25

Curiel, S., Raymond, J. C., Moran, J. M., Rodríguez, L. F., \& Cantó, J. 1990, ApJ, 365, L85

Draine, B. T., \& McKee, C. F. 1993, ARA\&A, 31, 373

Draine, B. T., Roberge, W. G., \& Dalgarno, A. 1983, ApJ, 264, 485

Dutrey, A., Guilloteau, S., \& Bachiller, R. 1997, A\&A, 325, 758

Flower, D. R., \& Pineau des Forets, G. 1994, MNRAS, 268, 724

Garay, G., Köhnenkamp, I., Bourke, T. L., Rodríguez, L. F., \& Lehtinen, K. K. 1998, ApJ, 509, 768

Garay, G., Mardones, D., Rodríguez, L. F., Caselli, P., \& Bourke, T. L. 2002, ApJ, 567, 980

Geppert, W. D., Hamberg, M., Thomas, R. D., et al. 2006, Faraday Discussions, 133,177

Girart, J. M., \& Acord, J. M. P. 2001, ApJ, 552, L63

Glassgold, A. E., Mamon, G. A., \& Huggins, P. J. 1991, ApJ, 373, 254

Goldsmith, P. F., \& Langer, W. D. 1999, ApJ, 517, 209

Greve, A., Kramer, C., \& Wild, W. 1998, A\&AS, 133, 271

Gusdorf, A., Cabrit, S., Flower, D. R., \& Pineau Des Forêts, G. 2008a, A\&A, 482,809

Gusdorf, A., Pineau Des Forêts, G., Cabrit, S., \& Flower, D. R. 2008b, A\&A, 490, 695

Hirano, N., \& Taniguchi, Y. 2001, ApJ, 550, L219

Hirano, N., Ho, P. P. T., Liu, S.-Y., et al. 2010, ApJ, 717, 58

Hollenbach, D., \& McKee, C. F. 1980, ApJ, 241, L47

Jiménez-Serra, I., Caselli, P., Martín-Pintado, J., \& Hartquist, T. W. 2008, A\&A, 482,549

Jørgensen, J. K., Bourke, T. L., Myers, P. C., et al. 2007, ApJ, 659, 479

Langer, W. D., \& Penzias, A. A. 1990, ApJ, 357, 477

Le Bourlot, J., Pineau des Forêts, G., Flower, D. R., \& Cabrit, S. 2002, MNRAS, 332,985

Lee, C.-F., Hasegawa, T. I., Hirano, N., et al. 2010, ApJ, 713, 731

Machida, M. N., Inutsuka, S.-I., \& Matsumoto, T. 2008, ApJ, 676, 1088

Maury, A. J., André, Ph., Hennebelle, P., et al. 2010, A\&A, 512, A40

Margulis, M., \& Lada, C. J. 1985, ApJ, 299, 925

Moriarty-Schieven, G. H., Snell, R. L., Strom, S. E., et al. 1987, ApJ, 319, 742

Müller, H. S. P., Thorwirth, S., Roth, D. A., \& Winnewisser, G. 2001, A\&A, 370, L49

Müller, H. S. P., Schlöder, F., Stutzki, J., \& Winnewisser, G. 2005, J. Mol. Struct., 742,215

Neufeld, D. A., \& Dalgarno, A. 1989, ApJ, 340, 869

Nisini, B., Codella, C., Giannini, T., et al. 2007, A\&A, 462, 163

Panoglou, D., Cabrit, S., Garcia, P. J. V., \& Forêts, G. P. D. 2009, in Protostellar Jets in Context, 459

Pineau des Forêts, G., Roueff, E., \& Flower, D. R. 1990, MNRAS, 244, 668

Pineau des Forêts, G., Roueff, E., Schilke, P., \& Flower, D. R. 1993, MNRAS, 262, 915

Raga, A., \& Cabrit, S. 1993, A\&A, 278, 267

Raga, A. C., Binette, L., Canto, J., \& Calvet, N. 1990, ApJ, 364, 601

Reipurth, B., \& Bally, J. 2001, ARA\&A, 39, 403

Richer, J. S., Hills, R. E., \& Padman, R. 1992, MNRAS, 254, 525

Rodríguez-Fernández, N., Tafalla, M., Gueth, F., \& Bachiller, R. 2010, A\&A, 516, A98

Santiago-García, J., Tafalla, M., Johnstone, D., \& Bachiller, R. 2009, A\&A, 495, 169

Schilke, P., Walmsley, C. M., Pineau des Forets, G., \& Flower, D. R. 1997, A\&A, 321,293

Shang, H., Allen, A., Li, Z.-Y., et al. 2006, ApJ, 649, 845

Tafalla, M., Myers, P. C., Caselli, P., \& Walmsley, C. M. 2004a, A\&A, 416, 191 Tafalla, M., Santiago, J., Johnstone, D., \& Bachiller, R. 2004b, A\&A, 423, L21

Tafalla, M., Santiago-García, J., Myers, P. C., et al. 2006, A\&A, 455, 577

Tielens, A. G. G. M., McKee, C. F., Seab, C. G., \& Hollenbach, D. J. 1994, ApJ, 431, 321

Tomida, K., Tomisaka, K., Matsumoto, T., et al. 2010, ApJ, 714, L58

Umemoto, T., Iwata, T., Fukui, Y., et al. 1992, ApJ, 392, L83

van Dishoeck, E. F., \& Blake, G. A. 1998, ARA\&A, 36, 317

Zapata, L. A., Schmid-Burgk, J., Muders, D., et al. 2010, A\&A, 510, A2

Ziurys, L. M., Friberg, P., \& Irvine, W. M. 1989, ApJ, 343, 201

Watanabe, N., Nagaoka, A., Shiraki, T., \& Kouchi, A. 2004, ApJ, 616, 638

Pages 17 to 19 are available in the electronic edition of the journal at http://www . aanda. org 


\section{Appendix A: A survey of EHV SO emission in 104166}

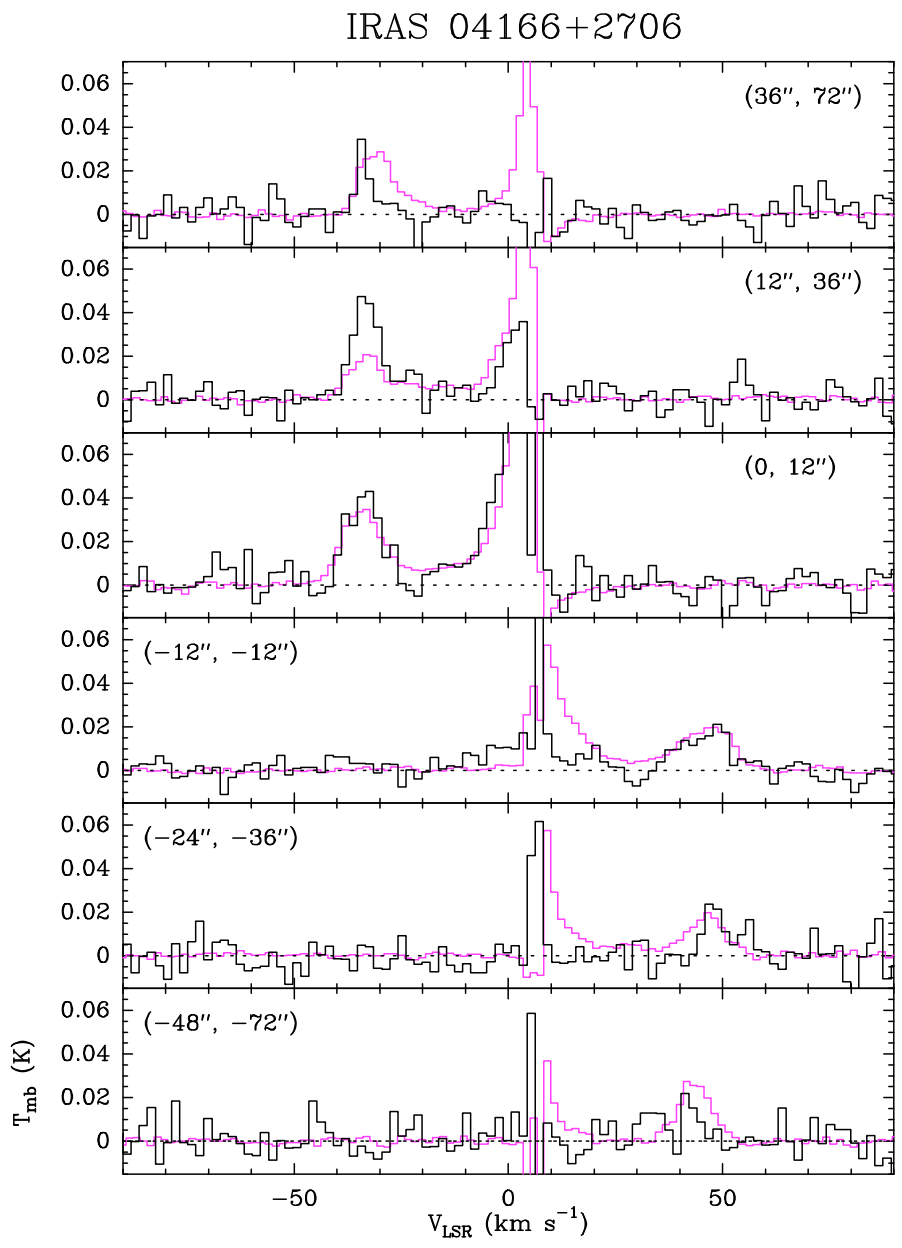

Fig. A.1. $\mathrm{SO}\left(3_{2}-2_{1}\right)$ (black) and $\mathrm{CO}(2-1)$ (red) spectra along the central axis of the I04166 outflow. The CO spectra have been scaled down by a factor of 20 , and the offsets are referred to the IRAS nominal position $\left(\alpha(\mathrm{J} 2000)=4^{\mathrm{h}} 19^{\mathrm{m}} 42^{\mathrm{s}} \cdot 6, \delta(J 2000)=+27^{\circ} 13^{\prime} 38^{\prime \prime}\right)$.

The detection of relatively strong SO emission in the EHV component of both L1448 and I04166 target positions was unexpected. No SO emission from EHV gas had previously been reported, so no information on its distribution and relation to other EHV tracers like $\mathrm{CO}$ and $\mathrm{SiO}$ was available. To better characterize this $\mathrm{SO}$ emission, we made small maps around both sources. In I04166, we observed 6 positions along the outflow axis and in L1448 we observed three positions. This appendix presents the I04166 data (the more limited L1448 survey is consistent with the I04166 results).

Figure A. 1 shows the $\mathrm{SO}\left(3_{2}-2_{1}\right)$ and $\mathrm{CO}(2-1)$ spectra observed simultaneously along the I04166 outflow axis. As can be seen, EHV SO emission was detected in at least 5 of the 6 target positions, both towards the red and blue lobes. Considering the limited $S / N$ of the data and the different beam size of the two transitions (24" in $\mathrm{SO}\left(3_{2}-2_{1}\right)$ and $11^{\prime \prime}$ in $\mathrm{CO}(2-1)$ ), we find a good match between the $\mathrm{SO}$ and $\mathrm{CO}$ data, indicating that the two EHV emissions originate from the same gas. In this respect, we can consider the position selected for the molecular survey as a likely representative of the outflow as a whole.

To further characterize the EHV SO emission in the I04166 outflow, we study the ratio between the $\mathrm{CO}(2-1)$ and $\mathrm{SO}\left(3_{2}-2_{1}\right)$
Table A.1. EHV emission along the I04166 outflow ${ }^{1}$.

\begin{tabular}{ccc}
\hline \hline $\begin{array}{c}\text { Offsets }^{2} \\
\left({ }^{\prime \prime},{ }^{\prime \prime}\right)\end{array}$ & $\begin{array}{c}I[\mathrm{CO}(2-1)]^{3} \\
\left(\mathrm{~K} \mathrm{~km} \mathrm{~s}^{-1}\right)\end{array}$ & $\begin{array}{c}I\left[\mathrm{SO}\left(3_{2}-2_{1}\right)\right]^{3} \\
\left(\mathrm{~K} \mathrm{~km} \mathrm{~s}^{-1}\right)\end{array}$ \\
\hline$(36,72)$ & 5.0 & 0.12 \\
$(12,36)$ & 3.9 & 0.38 \\
$(0,12)$ & 7.7 & 0.40 \\
$(-12,-12)$ & 5.2 & 0.21 \\
$(-24,-36)$ & 3.9 & 0.13 \\
$(-48,-72)$ & 4.9 & 0.07 \\
\hline
\end{tabular}

Notes. ${ }^{(1)}$ Velocity ranges are $[-43,-26]$ for blue EHV and $[37,54]$ for red EHV; (2) offsets as in Fig. A.1; (3) typical $\sigma(\mathrm{I})$ is $0.1 \mathrm{~K} \mathrm{~km} \mathrm{~s}^{-1}$ for $\mathrm{CO}(2-1)$ and $0.03 \mathrm{~K} \mathrm{~km} \mathrm{~s}^{-1}$ for $\mathrm{SO}\left(3_{2}-2_{1}\right)$.

integrated intensities. Table A.1 presents the values measured in our survey using the LSR velocity ranges -43 to $-26 \mathrm{~km} \mathrm{~s}^{-1}$ for the blue EHV gas and 37 to $54 \mathrm{~km} \mathrm{~s}^{-1}$ for the red EHV gas. As can be seen, the $\mathrm{CO}(2-1) / \mathrm{SO}\left(3_{2}-2_{1}\right)$ ratio in the innermost 4 positions is on the order of 20 , which is consistent with the value derived in the molecular survey of Sect. 3 . In the outermost position of each outflow lobe, however, the $\mathrm{SO}\left(3_{2}-2_{1}\right)$ emission weakens significantly, and the $\mathrm{CO}(2-1) / \mathrm{SO}\left(3_{2}-2_{1}\right)$ ratio increases to about 40 towards the northern (blue) lobe and twice as much towards the southern (red) lobe.

The close to constant $\mathrm{CO} / \mathrm{SO}$ intensity ratio along the inner EHV jet suggests that both the SO excitation and the abundance relative to $\mathrm{CO}$ are close to constant for this outflow component over the central $\approx 0.03 \mathrm{pc}$, and that they have values similar to those derived for the $\left(8^{\prime \prime}, 14^{\prime \prime}\right)$ position, for which additional transitions of both species were observed. The increase of the $\mathrm{CO} / \mathrm{SO}$ intensity ratio in the outer outflow, on the other hand, suggests that there is a drop in the SO abundance or excitation of the EHV gas about 0.05 pc from the outflow source. Lacking additional SO data, we use the $\mathrm{CO}$ and $\mathrm{SiO}$ observations of Santiago-García et al. (2009) to investigate this drop. The highresolution $\mathrm{CO}$ and $\mathrm{SiO}$ interferometric observations showed a similar factor of 2-3 increase in the $\mathrm{CO}(2-1) / \mathrm{SiO}(2-1)$ ratio in the EHV component as a function of distance from the protostar. The interferometric data, in addition, showed that both the $\mathrm{CO}$ and $\mathrm{SiO}$ emitting regions broaden with distance to the YSO, suggesting that the EHV gas is spreading laterally as it moves away from the protostar (in agreement with models of internal working surfaces, Dutrey et al. 1997; Santiago-García et al. 2009). In this case, it seems therefore likely that the excitation of $\mathrm{SiO}$ drops faster than that of $\mathrm{CO}$ due to its higher dipole moment, and that the observed change in the $\mathrm{CO} / \mathrm{SiO}$ ratio is at least in part produced by a change in excitation. As the SO molecule has also a high dipole moment, it is very likely that the increase in the $\mathrm{CO} / \mathrm{SO}$ ratio with distance from I04166 also results, at least in part, from a change in excitation. Multitransition observations are required to fully constrain the characteristics of the EHV gas in I04166.

Although our observations seem to represent the first detection of SO in EHV gas, bright SO emission in the jet-like components of the HH211 and Ori-S6 outflows have been recently reported by Lee et al. (2010) and Zapata et al. (2010), respectively. The jet-like component of these outflows has significantly lower velocity than the components in L1448 and I04166, and their emission does not form distinct EHV features in the $\mathrm{CO}$ spectra like those shown in Fig. 2. These jet-like components, however, may represent a similar phenomenon to the EHV gas, and may owe their low radial velocity just to a location close to 
the plane of the sky. Lacking accurate measurements of outflow inclination angles, a comparison between the chemical properties of these objects may help test the idea that all the jetlike components share a common physics (and likely origin). If so, chemical composition may result a more discriminant tracer of outflow properties than gas kinematics, and the presence of EHV gas in outflows may be more common among Class 0 sources than currently recognized.

\section{Appendix B: Correction for differential beam dilution}

The beam size of a diffraction-limited telescope like the IRAM $30 \mathrm{~m}$ depends linearly on the operating wavelength, so for a given molecule, transitions having different rest frequencies are observed using different angular resolutions. At the $\mathrm{CO}(1-0)$ frequency, for example, the $F W H M$ of the $30 \mathrm{~m}$ main beam is about 21 arcsec, while the main beam size for $\mathrm{CO}(2-1)$ is half that value (Greve et al. 1998). This significant change in the beam size with frequency, together with the small angular size of the regions under study, makes each transition in our survey suffer from a different amount of beam dilution. Such a differential effect needs to be corrected before using the observed intensities to derive molecular abundances, or otherwise the high frequency (small beam) transitions will be over weighted, producing an overestimate of both the molecular excitation and the species column density. In this appendix we present the method we have used to compensate for this common observational problem.

Properly correcting all our survey data for the effects of beam dilution would require making high angular resolution maps for each transition. In the absence of such data, we used observations of the main outflow tracer, $\mathrm{CO}$, to characterize the distribution of gas in the vicinity of our survey targets, and to estimate how the observed spectrum depends on the beam size. To this end, we made a Nyquist-sampled map around each outflow target in $\mathrm{CO}(2-1)$, as this transition provides an effective angular resolution higher than most transitions in our survey $\left(\approx 11^{\prime \prime}\right)$. This $\mathrm{CO}(2-1)$ map was used to simulate observations with larger beam sizes by convolving the Nyquist-sampled spectra to the required resolution. As a result, we produced a grid of $\mathrm{CO}(2-1)$ spectra with effective beam sizes ranging from about 12" (close to the beam size for $\mathrm{CO}(2-1)$ ) to about $30^{\prime \prime}$ (larger than the largest beam in our survey). To double check the I04166 results (where a pointing error was suspected in the Nyquist map), we repeated the calculation using the combined Plateau de Bure$30 \mathrm{~m}$ data set from Santiago-García et al. (2009), which contains all the spatial frequencies, is independent of our new $30 \mathrm{~m}$ Nyquist-sampled map, and has an angular resolution of about $3^{\prime \prime}$.

The results of our convolution analysis are presented in Fig. B.1 in the form of the $\mathrm{CO}(2-1)$ integrated intensities as a function of the total convolving beam size for the three outflow regimes defined in the molecular survey. As can be seen, the intensity in all regimes drops systematically with beam size, which is an indication that the emission is only partly resolved by the telescope, and that differential beam dilution truly affects our survey data. As can also be seen, the slope of the drop is different in each outflow regime, and it steepens systematically from the s-wing to the EHV gas. This steepening is in good agreement with the previous observation that outflow collimation increases systematically with velocity in both L1448 and I04166 (Bachiller et al. 1990; Tafalla et al. 2004b). Also seen in Fig. B.1 is the good agreement between the two data sets of I04166 for beam sizes larger than about $14^{\prime \prime}$. The slight disagreement for smaller beam sizes partly results from the high noise in the $30 \mathrm{~m}$

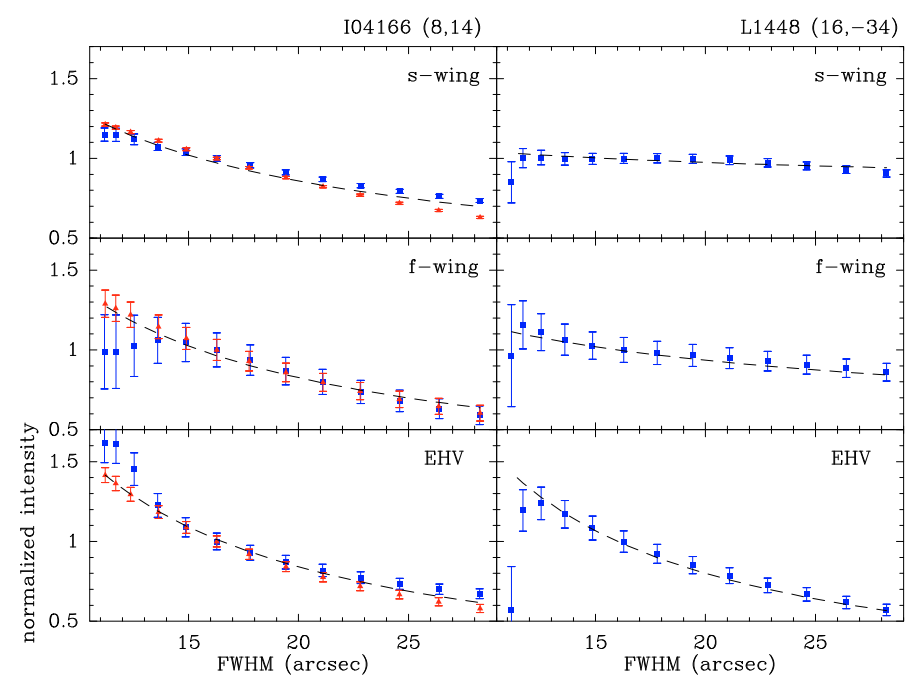

Fig. B.1. Variation of the $\mathrm{CO}(2-1)$ integrated intensity as a function of total convolving beam size (normalized to the $16^{\prime \prime}$ value) for the three outflow velocity regimes used in the molecular survey. The intensities have been determined by convolving appropriately either IRAM $30 \mathrm{~m}$ Nyquist-sampled maps (blue squares) or a combined Plateau de Bure interferometer $+30 \mathrm{~m}$ data set (red triangles). The dashed lines represent a set of fits with the form $\Omega_{\mathrm{MB}}^{-\alpha}$ that have been used to correct the survey data for beam dilution effects (see text). The error bars represent the estimated $1-\sigma \mathrm{rms}$ and have been calculated from the noise in the spectra using a baseline fit to the line-free channels.

spectra with small beam size, as the Nyquist-sampled map is relatively shallow and only a few spectra contribute to the small beam size data points. This small beam size regime is also very sensitive to small pointing errors, and this seems to be the cause of some deviations seen in both the I04166 and L1448 plots.

The systematic dependence of the integrated intensity with observing beam size shown in Fig. B.1 suggests that the effect of beam dilution can be corrected, at least to first order, using a simple parameterization. For a symmetric Gaussian beam, the beam dilution factor is proportional to $\Omega_{\mathrm{MB}}^{-2}$ if the source is point like, to $\Omega_{\mathrm{MB}}^{-1}$ if the source one dimensional, and trivially equal to $\Omega_{\mathrm{MB}}^{0}$ if the source is infinitely extended in two dimensions. It seems therefore reasonable that when fitting the curves of Fig. B.1, we use an expression of the form $\Omega_{\mathrm{MB}}^{-\alpha}$, where $\alpha$ is a free parameter expected to lie between 0 and 2 . This expectation is confirmed by the data, and as the dashed curves in Fig. B.1 show, the analytic expression fits the data well inside the range of observations. The $\alpha$ coefficient in the fit is $0.6,0.75$, and 0.9 for the s-wing, f-wing, and EHV ranges of I04166, and 0.1, 0.3, and 1.0 for the same ranges in L1448, respectively. Of particular interest is the close-to-1 values derived for the EHV range in both outflows, a further indication that this fastest outflow regime is highly collimated.

With the above $\Omega_{\mathrm{MB}}^{-\alpha}$ fits we have converted all measured integrated intensities into equivalent values for a $16^{\prime \prime}$ beam, which is the average size of the beams used in in our survey. The excitation and column density estimates presented in this paper therefore represent estimates of these parameters inside a $16^{\prime \prime}$ diameter beam.

\section{Appendix C: Fitting multiple-component spectra with templates}

The spectra of $\mathrm{HCN}$ and $\mathrm{CH}_{3} \mathrm{OH}$ contain multiple components that partly overlap, and to calculate the integrated intensity of 
each of these components in each outflow regime, we need a method to disentangle them. An inspection of the data shows that in each multi-component spectrum, all components present similar shape and differ only in velocity and relative intensity, and this suggests that it should be possible to disentangle the outflow contributions by fitting the complex spectrum with a synthetic one made of multiple copies of the same line profile. To carry out such a fitting procedure, we have taken spectra from single lines (from $\mathrm{CO}, \mathrm{SO}, \mathrm{CS}$, or $\mathrm{SiO}$ ) as templates, made copies of them using the CLASS software, and shifted each template in frequency by the appropriate value for each component (as provided by the Cologne Database for Molecular Spectroscopy, see Müller et al. 2001, 2005). By varying the relative intensity of each template, which is the only free parameter in the procedure, we have fitted the multi-component spectra, and from this fit, we estimate the integrated intensity of each line component in the three outflow regimes (only the outflow part was fitted while the often optically thick ambient emission was ignored).

To identify the best fit solution, we selected a set of velocity ranges in the spectrum where the overlap between components is minor or where one component clearly dominates the emission, and we required that the synthetic spectrum matched the observed integrated intensity within the noise level. For the case of $\mathrm{HCN}(1-0)$, we found that the three hyperfine components had relative intensities very close to their statistical weights, suggesting that the outflow emission is optically thin and that the excitation of the sublevels is close to LTE. By fixing the relative intensities of the components to their statistical weights, the only one free parameter left to fit the observed emission profile was a global scaling factor.

As shown in Fig. 4, different species present slightly different outflow components, reflecting their peculiar abundance pattern across the outflow regimes (Sect. 4.2). For this reason, some species provide better templates than others to fit a given multicomponent spectrum, and finding the best match is necessary a matter of trial and error. To test how sensitively the final integrated intensities depend on the choice of template species, we fitted the HCN(1-0) line from L1448 (the multiple-component spectrum with highest $S / N$ in our sample) using templates from $\mathrm{CS}(2-1), \mathrm{SO}\left(3_{2}-2_{1}\right), \mathrm{CO}(2-1)$, and $\mathrm{SiO}(2-1)$. When these templates are properly scaled to match the observed spectrum, the rms dispersion in the estimate of the outflow contribution to the s-wing, f-wing, and EHV regimes is $12 \%, 2 \%$, and 14\%, respectively. If these dispersions are estimates of the uncertainty introduced by the multi-component fitting procedure, we conclude that the added uncertainty is comparable to the uncertainty in the telescope calibration and in the correction for beam dilution. The template method, therefore, seems to provide an accurate way to estimate the outflow contribution in multiple-component profiles.

\section{Appendix D: Revised abundances for L1157-B1}

To properly compare the abundances of our target outflows with those of the L1157-B1 position, often considered as a standard in outflow chemistry, we have re-evaluated the L1157-B1 estimates of Bachiller \& Pérez Gutiérrez (1997) for the species detected in both L1448 and I04166. We have used the original
Table D.1. Molecular column densities and excitation temperatures for L1157-B1.

\begin{tabular}{lcccc}
\hline \hline & \multicolumn{2}{c}{ s-wing $^{1}$} & \multicolumn{2}{c}{ f-wing } \\
MOLEC & $\begin{array}{c}N_{T} \\
\left(\mathrm{~cm}^{-2}\right)\end{array}$ & $\begin{array}{c}T_{\text {ex }} \\
(\mathrm{K})\end{array}$ & $\begin{array}{c}N_{T} \\
\left(\mathrm{~cm}^{-2}\right)\end{array}$ & $\begin{array}{c}T_{\text {ex }} \\
(\mathrm{K})\end{array}$ \\
\hline $\mathrm{CO}$ & $3.2 \times 10^{16}$ & 51 & $1.1 \times 10^{16}$ & 52 \\
$\mathrm{SiO}$ & $1.9 \times 10^{13}$ & 12 & $1.1 \times 10^{13}$ & 12 \\
$\mathrm{SO}$ & $8.1 \times 10^{13}$ & 17 & $2.3 \times 10^{13}$ & 15 \\
$\mathrm{CS}$ & $4.8 \times 10^{13}$ & 10 & $1.7 \times 10^{13}$ & 12 \\
$\mathrm{CH}_{3} \mathrm{OH}^{2}$ & $1.0 \times 10^{15}$ & 11 & $2.1 \times 10^{14}$ & 11 \\
$\mathrm{HC}_{3} \mathrm{~N}$ & $8.2 \times 10^{12}$ & 26 & $2.5 \times 10^{12}$ & 23 \\
$\mathrm{HCO}^{+}$ & $5.3 \times 10^{12}$ & $12^{3}$ & $1.7 \times 10^{12}$ & $12^{3}$ \\
$\mathrm{o}-\mathrm{H}_{2} \mathrm{CO}$ & $6.4 \times 10^{13}$ & $12^{3}$ & $1.6 \times 10^{13}$ & $12^{3}$ \\
$\mathrm{HCN}$ & $9.8 \times 10^{13}$ & $12^{3}$ & $2.9 \times 10^{13}$ & $12^{3}$ \\
\hline
\end{tabular}

Notes. ${ }^{(1)} V_{\text {LSR }}$ limits are -3.5 to $0.5 \mathrm{~km} \mathrm{~s}^{-1}$ for s-wing and -7.5 to $-3.5 \mathrm{~km} \mathrm{~s}^{-1}$ for $\mathrm{f}$-wing; (2) The $\mathrm{E}$ and $\mathrm{A}$-forms of $\mathrm{CH}_{3} \mathrm{OH}$ were analyzed separately and the column density in the table is the sum of the two results. The $\mathrm{E} / \mathrm{A}$ ratio was found to $\mathrm{be} \approx 1.2$ in both outflow regimes; (3) $T_{\mathrm{ex}}=12 \mathrm{~K}$ was assumed.

data from Bachiller \& Pérez Gutiérrez (1997) and we have applied to it the same procedure used in the analysis of L1448 and I04166: correction for beam dilution, use of templates to disentangle overlapping components, and use of population diagram analysis for species with more than one detected transition. In contrast with Bachiller \& Pérez Gutiérrez (1997), who integrated the intensities across the full line profile, we have ignored LSR velocities less than $1.8 \mathrm{~km} \mathrm{~s}^{-1}$ away from the systemic velocity $\left(V_{\mathrm{LSR}}=2.3 \mathrm{~km} \mathrm{~s}^{-1}\right)$ to avoid possible contamination from the ambient cloud. This velocity limit was set by the point at which the ${ }^{13} \mathrm{CO}(2-1)$ emission drops to the noise level in the spectrum. As in L1448 and I04166, we have divided the line wing into two regimes that will be referred to as the s-wing and the f-wing (see limits in Table D.1). For each outflow regime, we have derived beam-dependent dilution factors as described in Appendix B, using the CO(2-1) data from Bachiller \& Pérez Gutiérrez (1997), and we have fitted the results with power laws having $\alpha$ values of 0.6 and 0.7 for s-wing and f-wing, respectively.

The results of the abundance re-analysis are presented in Table D.1. To better determine the relatively high $T_{\mathrm{ex}}$ value of CO $(\approx 50 \mathrm{~K})$, we have complemented the $J=1-0$ and $2-1$ data of Bachiller \& Pérez Gutiérrez (1997) with the 3-2, 4-3, and 6-5 data from Hirano \& Taniguchi (2001), also corrected for beam-dilution effects. For species with only one observed transition, we have used a default $T_{\mathrm{ex}}$ value of $12 \mathrm{~K}$ as determined from the population diagram analysis of $\mathrm{SiO}, \mathrm{CS}$, and SO. Overall, the results of our re-analysis are in good (factor of 2) agreement with those of Bachiller \& Pérez Gutiérrez (1997) when the abundances are normalized to that of CO. A comparison between the values for the s-wing and f-wing regimes shows that, as in L1448 and $\mathrm{I} 04166, \mathrm{SiO}$ becomes relatively more abundant at high velocities, while $\mathrm{CH}_{3} \mathrm{OH}$ and $\mathrm{H}_{2} \mathrm{CO}$ decrease in abundance with velocity. The other species re-analyzed here present similar abundances within $20 \%$ in the two outflow regimes. 